\title{
Acoustic Measurements of a Large Civil Transport Main Landing Gear Model
}

\author{
Patricio A. Ravetta ${ }^{1}$ \\ AVEC Inc., Blacksburg, Virginia 24060 \\ Mehdi R. Khorrami ${ }^{2}$ \\ NASA Langley Research Center, Hampton, Virginia, 23681 \\ Ricardo A. Burdisso ${ }^{3}$, and David M. Wisda ${ }^{4}$ \\ AVEC Inc., Blacksburg, Virginia 24060
}

\begin{abstract}
Microphone phased array acoustic measurements of a 26\%-scale, Boeing 777-200 main landing gear model with and without noise reduction fairings installed were obtained in the anechoic configuration of the Virginia Tech Stability Tunnel. Data were acquired at Mach numbers of $0.12,0.15$, and 0.17 with the latter speed used as the nominal test condition. The fully and partially dressed gear with the truck angle set at $13^{\circ}$ toe up landing configuration were the two most extensively tested configurations, serving as the baselines for comparison purposes. Acoustic measurements were also acquired for the same two baseline configurations with the truck angle set at $0^{\circ}$. In addition, a previously tested noise reducing, toboggan-shaped fairing was re-evaluated extensively to address some of the lingering questions regarding the extent of acoustic benefit achievable with this device. The integrated spectra generated from the acoustic source maps reconfirm, in general terms, the previously reported noise reduction performance of the toboggan fairing as installed on an isolated gear. With the recent improvements to the Virginia Tech tunnel acoustic quality and microphone array capabilities, the present measurements provide an additional, higher quality database to the acoustic information available for this gear model.
\end{abstract}

\section{Introduction}

A irframe borne noise is a significant component of aircraft noise during landing when the engines are at low power settings, the wing high-lift devices such as slats and flaps are deflected, and the landing gear is deployed. ${ }^{1-6}$ For medium and large size civil transports, the noise generated by the undercarriage system, especially the main landing gear, constitutes a major portion of the airframe noise. ${ }^{1,4-6}$ Development and advancement of system-level, simulationbased airframe noise prediction methodologies is being pursued under the NASA Environmentally Responsible Aviation (ERA) project. Given the daunting geometrical complexities associated with the main gear of large civil aircraft, accurate prediction of the noise generated by such a structure via high-fidelity simulations, even on a component level basis, has remained elusive. The present effort is an attempt to generate a high-quality acoustic database that can serve the dual purpose of providing a deeper understanding of the various acoustic sources associated with the main gear structures as well as validating the ongoing simulation-based airframe noise prediction work.

Large scale, system-level simulations of model- and full-scale aircraft accomplished under the NASA-Gulfstream partnership on airframe noise research have firmly established the utility of the computational approach as a powerful complementary tool to wind tunnel and flight testing with regard to the prediction and mitigation of airframe noise. ${ }^{7-}$

${ }^{12}$ The aforementioned simulations involved a business (regional) jet class of aircraft. Extension and application of a similar simulation approach to a large civil transport in landing configuration is an important goal of the NASA Aeronautics Research Mission Directorate. The achievement of this ambitious goal requires execution of several steps

${ }^{1}$ Chief Research Engineer, Senior Member AIAA.

${ }^{2}$ Aerospace Engineer, Computational AeroSciences Branch, Associate Fellow AIAA.

${ }^{3}$ President, Associate Fellow AIAA.

${ }^{4}$ Project Engineer. 
ranging from the selection of suitable geometries to gathering/generation of requisite experimental aeroacoustic data for validation purposes.

The 26\%-scale, Boeing 777-200 main landing gear model in isolation provides an ideal platform to commence the process. The high-fidelity model is representative of the most geometrically complex main landing gear systems flown on current large civil transports. The model has been extensively evaluated in previous test campaigns both in isolated, component-level configuration ${ }^{13-15}$ and as part of the 26\%-scale semi-span model of the 777-200 aircraft tested in the NASA Ames 40- by 80-ft wind tunnel. ${ }^{16}$ However, most of the previous isolated gear acoustic tests were executed in hard wall tunnels where close proximity of the microphone array to the model, combined with wall reflection effects, added a significant amount of uncertainty to the measured data. In addition, these earlier measurements were made with microphone arrays that were less capable when compared to today's optimized designs. Validation of the ongoing simulations for the 26\%-scale 777-200 main gear model necessitated the re-acquisition of model acoustic measurements under better test conditions. Recent improvements to the Virginia Tech wind tunnel both in terms of aeroacoustic quality ${ }^{17}$ and microphone array capabilities provided the appropriate incentives for the present test campaign. An additional motivation was the need to retest the $26 \%$-scale gear model with the noise reducing toboggan-shaped fairing to reconcile the acoustic performance of this device in isolation with full-scale flight data obtained during the Quiet Technology Demonstrator 2 (QTD2) test of $2005 .^{6}$

\section{Experimental Setup}

\section{A. Wind Tunnel Facility}

The experiments presented here were conducted at the Virginia Tech Stability Wind Tunnel shown in Figure 1. The facility is a continuous, closed circuit, single return, subsonic wind tunnel with a $7.3 \mathrm{~m}(24 \mathrm{ft})$ long test section of dimensions 1.83 by $1.83 \mathrm{~m}$ ( 6 by $6 \mathrm{ft})$. The tunnel is powered by a $600 \mathrm{hp} \mathrm{DC}$ motor driving a $4.26 \mathrm{~m}(14 \mathrm{ft})$ propeller providing a maximum speed of about $280 \mathrm{~km} / \mathrm{h}(255 \mathrm{ft} / \mathrm{s})$ for the empty wind tunnel, i.e., Mach number (M) of 0.23 . The tunnel, which can be operated either in hard wall or anechoic configuration, provides uniform flow throughout the test section and low turbulence intensity. For the present test campaign, the tunnel was used in its anechoic configuration. A schematic of the test section and anechoic chambers is shown in Figure 2. Pictures of the installed landing gear model and test section are shown in Figures 3 and 4.
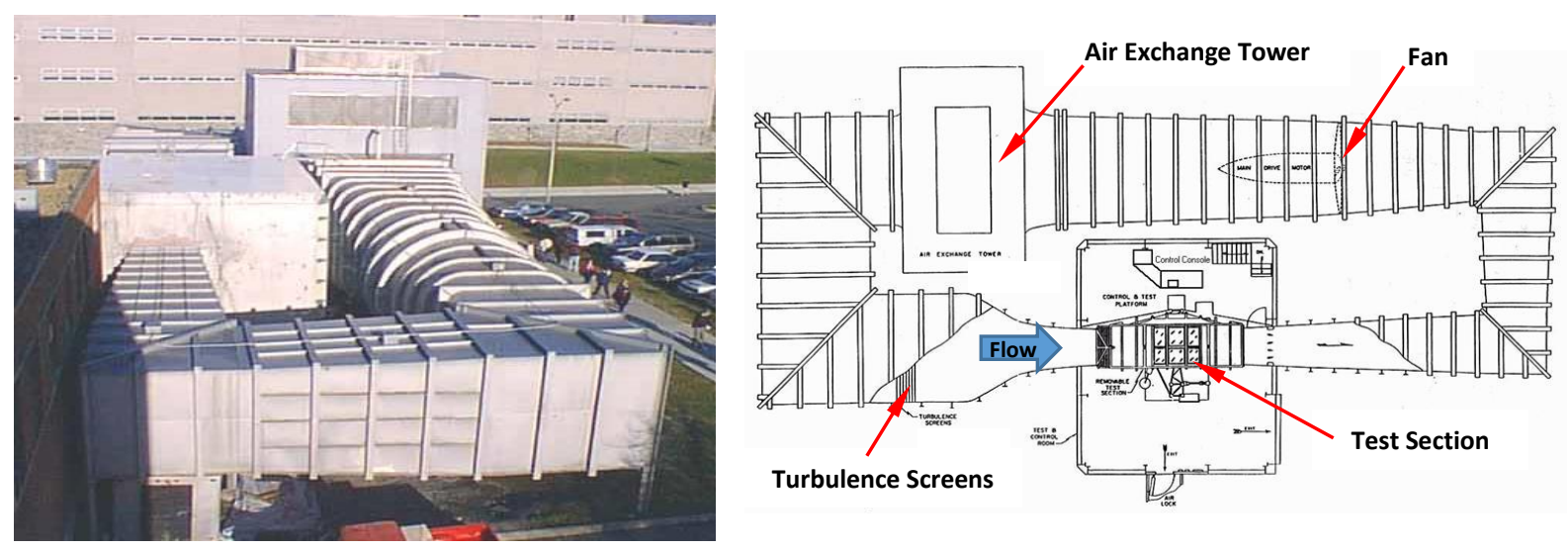

Figure 1. Picture and schematic of the Virginia Tech Stability Wind Tunnel.

The test section consists of acoustically treated upper and lower walls that run the full $7.3 \mathrm{~m}$ length of the test section and partial side walls, also treated, at the test section entrance and exit. Large rectangular openings in the side walls, which extend $5.14 \mathrm{~m}$ in the streamwise direction and cover the full $1.83 \mathrm{~m}$ height of the test section, serve as acoustic windows. Sound generated inside the tunnel circuit exits the test section through these acoustic windows into the anechoic chambers on either side. Large tensioned panels of Kevlar ${ }^{\circledR}$ cloth cover these openings, permitting the sound to pass while containing the bulk of the flow. The test section arrangement thus simulates a half-open jet, acoustically speaking. The Kevlar ${ }^{\circledR}$ windows eliminate the need for a jet catcher and, by containing the flow, substantially reduce the lift interference when airfoil models are tested. 
The upper (ceiling) and lower (floor) walls of the test section are constructed primarily from a series of perforated metal panels bonded to a layer of Kevlar ${ }^{\circledR}$ cloth that forms a smooth, quiet, but acoustically transparent flow surface. The volume behind this flow surface is filled with $0.457 \mathrm{~m}$ high foam wedges designed to eliminate any acoustic reflections at frequencies above $190 \mathrm{~Hz}$. Plain weave Kevlar $^{\circledR}$ is used to form the acoustic windows on the side walls. The cloth is stretched on 5.37 by $2.51 \mathrm{~m}$ tensioning frames. An anechoic chamber is positioned on each side of the test section. Both chambers have a streamwise length of $6 \mathrm{~m}$, extend $2.8 \mathrm{~m}$ out from the test section acoustic window, and have a height of $4.2 \mathrm{~m}$. The chamber walls are constructed from medium density fiberboard, supported by a network of external steel beams, and lined internally with $0.610 \mathrm{~m}$ high acoustic foam wedges. Quarter-elliptical foam sections surround the acoustic windows so as to form a smooth transition between the lower and upper walls of the test section on the inside of the windows and the acoustically treated walls of the anechoic chambers on the outside of the acoustic windows.

As shown in Figures 3 and 4, solid aluminum floor panels were used in the area surrounding the model mount for the sideline measurements. For the flyover measurements, the port side $\operatorname{Kevlar}^{\circledR}$ window was replaced by a solid wall on which the landing gear was mounted. This wall was constructed with aluminum honeycomb panels bolted to a wooden frame. In both setups, the region immediately surrounding the model was covered with a high-strength plywood panel reinforced with aluminum L-beams.

\section{B. Landing Gear Model}

The high fidelity, 26\%-scale, 777 main landing gear model used in this study was originally tested as part of the STAR (Subsonic Transport Aeroacoustic Research) model (a semi-span model of the 777) in the NASA Ames 40- by $80-\mathrm{ft}$ wind tunnel. ${ }^{16}$ The isolated gear model was also evaluated under the AST (Advance Subsonic Transport) and QAT (Quiet Aircraft Technology) programs in the NASA Ames 7- by 10-ft wind tunnel. ${ }^{13}$ This model was also extensively tested at Virginia Tech, both in the hard-wall configuration and an early version of the anechoic setup. ${ }^{14,15,17}$

The model installed in the Virginia Tech tunnel is shown in Figure 3a (baseline configuration) and Figure $3 \mathrm{~b}$ (toboggan fairing installed) in the actual landing position (e.g., the images have been rotated). For this work, the model was mounted on the test section floor for the sideline measurements

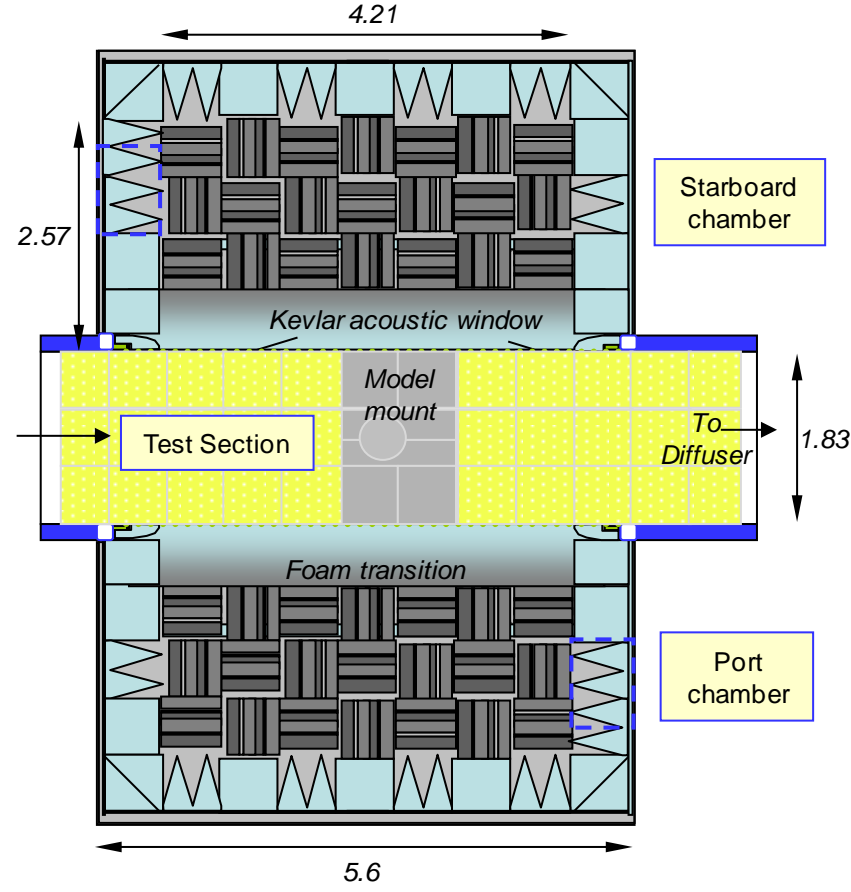

Figure 2. VT Stability Wind Tunnel Anechoic System. Cross-section through the anechoic test section and chambers as seen from above. Dimensions in meters.
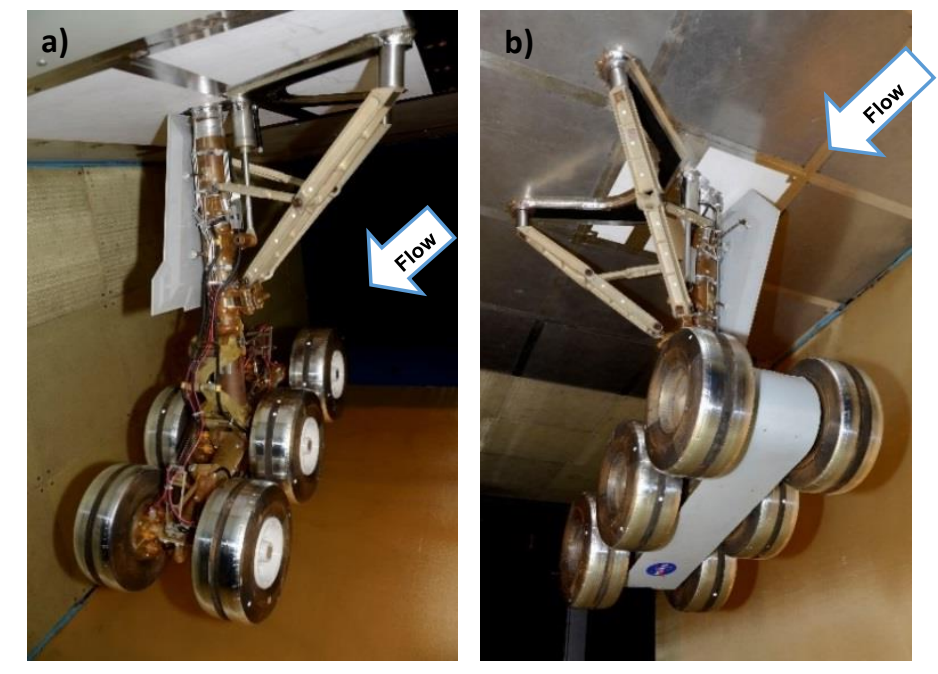

Figure 3. High fidelity $\mathbf{2 6 \%}$-scale 777 main landing gear model: a) baseline configuration and b) toboggan fairing installed. 
and on the port side wall for the flyover measurements, as shown in Figure 4. The high-fidelity model features all the major gear components: strut, braces, torque link, cable harnesses, lock links, main door, and wheels (see Figure 5 for naming convention of major components referred to throughout this manuscript). The model also includes most of the details found in the full-scale landing gear, such as oleo lines, cables, wheel hubs, brake cylinders, and hydraulic valves. The main structure of the model is made of steel and aluminum and the finer details (gear dressing) were mostly made in stereo lithography up to an accuracy of $3 \mathrm{~mm}$ in full-scale. The main differences with the actual landing gear are: the wheel hubs do not have the openings that allow air to flow freely through the wheels, a smaller door located close to the wing and attached to the main door is not in the model, and the wing cavity is not modeled.
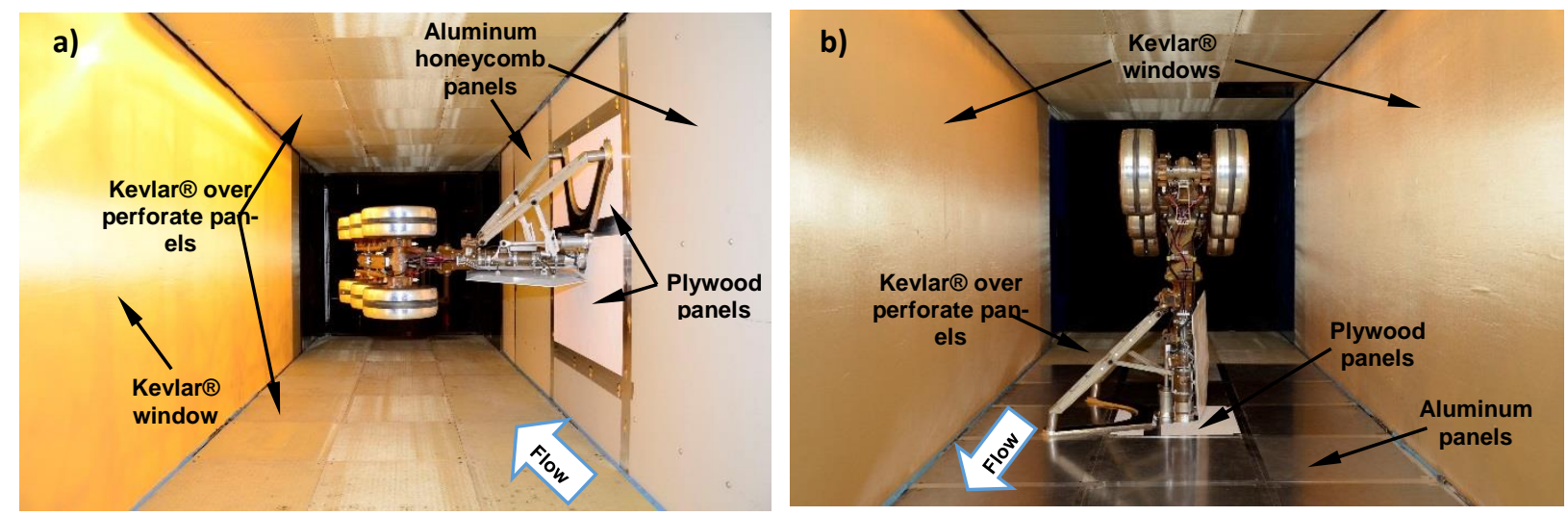

Figure 4. Landing gear model installed in the Virginia Tech anechoic wind tunnel test section for: a) flyover measurements (view from upstream), and b) sideline measurements (view from downstream).

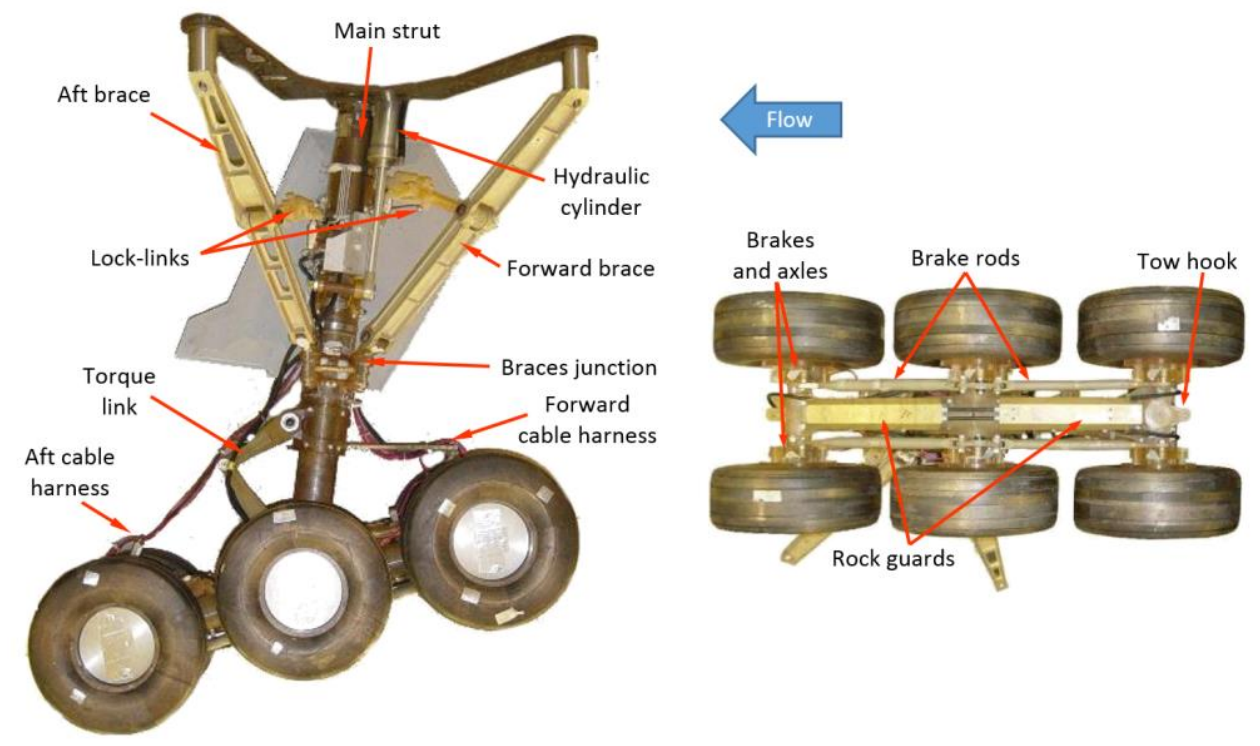

Figure 5. Schematic showing the names of major landing gear components.

\section{Toboggan Fairing}

The design of the flight-test toboggan was based on results for different toboggan configurations previously tested in the hard-wall configuration of the wind tunnel. ${ }^{15}$ The goal was to implement a fairing that would possess the attributes of the maximum width toboggan tested (and thus provide maximum noise reduction) while accounting for implementation issues in full scale, i.e., tire deflection and brake cooling effects. To this end, the minimum width toboggan (tested in 2007) was modified using a silicone elastomer with a polyester/fiberglass stiffening element to extend it as close as possible to the tires without compromising functionality. Schematics of the device and a picture of its installation on the model are shown in Figure 6. 

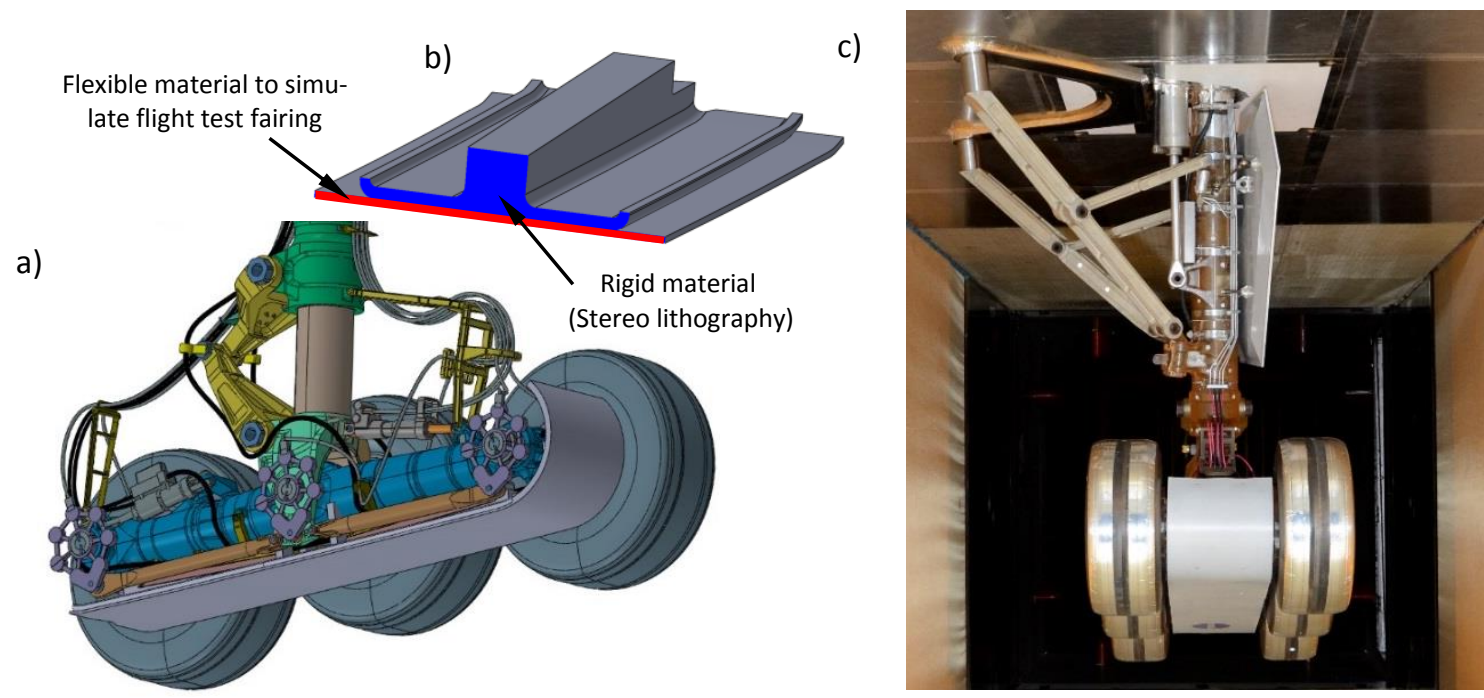

Figure 6. a) Section view of toboggan installed on landing gear, b) section view of toboggan fairing, and c) picture of toboggan configuration with truck at $13^{\circ}$ angle.

\section{Instrumentation}

The acoustic instrumentation used in this test consisted of two different microphone phased arrays recently developed and built by AVEC. All the flyover and sideline configurations were measured using a large array spanning most of the Kevlar ${ }^{\circledR}$ wall on the starboard side chamber. This array (shown in Figure 7a) consists of 251 GRAS type 40PH microphones with cabling, cable management, and signal conditioning systems custom built by AVEC. The $3.65 \times 1.75-\mathrm{m}$ array comprises four nested arrays (each designed as a non-redundant, seven-arm spiral array). One of the array design goals was to allow for directivity measurements along the tunnel streamwise direction. Another goal was to have a very large aperture to measure low frequency noise. For this reason, the full array was also optimized to minimize redundancy, with a resulting figure of merit of 0.994 . The array design, with the microphones in each of the four nested arrays color coded, is shown in Figure 8. Schematics of the position of the array relative to the landing gear model, test section, and Kevlar ${ }^{\circledR}$ window are shown in Figure 9.
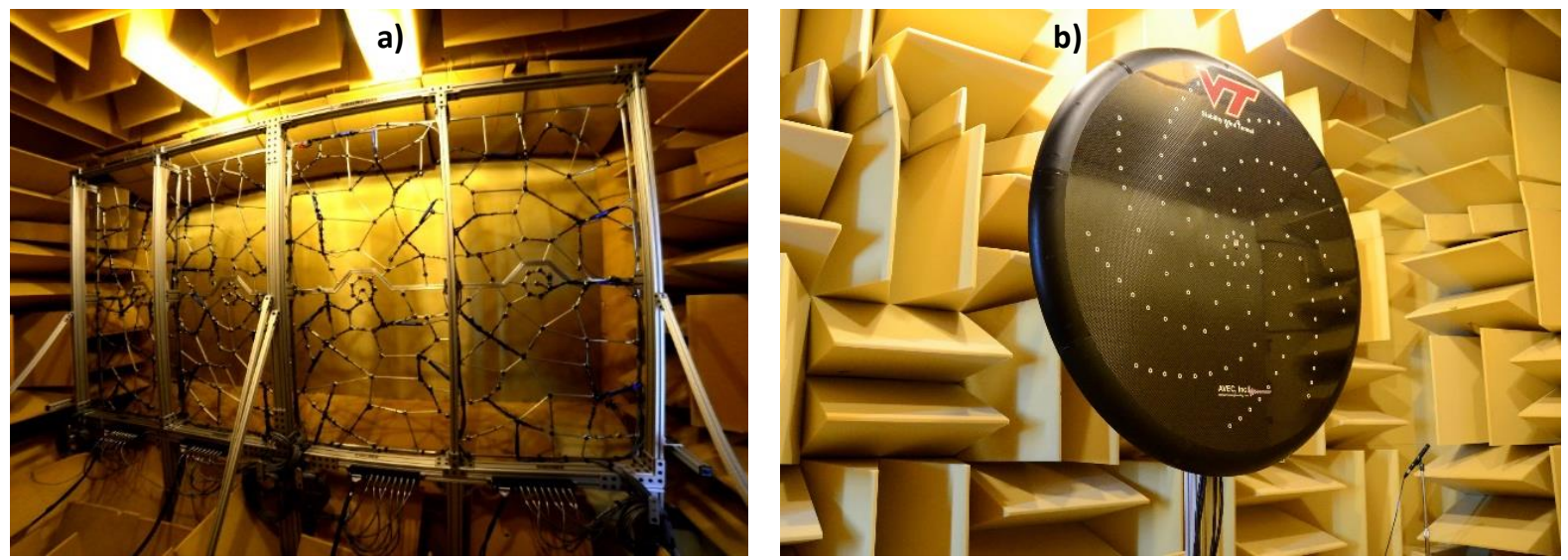

Figure 7. Pictures of a) 251-element microphone phased array installed in the starboard side anechoic chamber, and b) 117-element microphone phased array installed in the port side anechoic chamber.

For a subset of the sideline measurements, an additional 1.1-m diameter 117-element array (shown in Figure 7b) was installed in the port chamber. The 117 microphones in the array are arranged in a nine-arm spiral of 13 microphones each with sensor spacings determined using a proprietary array design code. The microphones used in this array are Panasonic WM-64PNT Electret microphones. These microphones have a flat frequency response from 20$16,000 \mathrm{~Hz}$ and a nominal sensitivity of $-44+/-3 \mathrm{~dB}$ referenced to $1 \mathrm{~V} / \mathrm{Pa}$ at $1,000 \mathrm{~Hz}$. All the cartridges in the array were calibrated before assembly and selected to be within $\pm 5^{\circ}$ phase and $\pm 0.4 \mathrm{~dB}$ amplitude from 500 to $16,000 \mathrm{~Hz}$. 
The signals for all GRAS microphones were routed through two AVEC 128-channel IEPE signal conditioning and anti-aliasing filter systems. The data for all channels were acquired simultaneously for 32 seconds at a sampling rate of $51,200 \mathrm{~Hz}$ using General Standards cards installed in a custombuilt computer controlled by AVEC's Phased Array software. Configurations in which the 117-element array was also used, an additional (non-IEPE) system with 128 channels was synchronized (for simultaneous sampling) with the system for the large array.

a) Flyover measurements setup
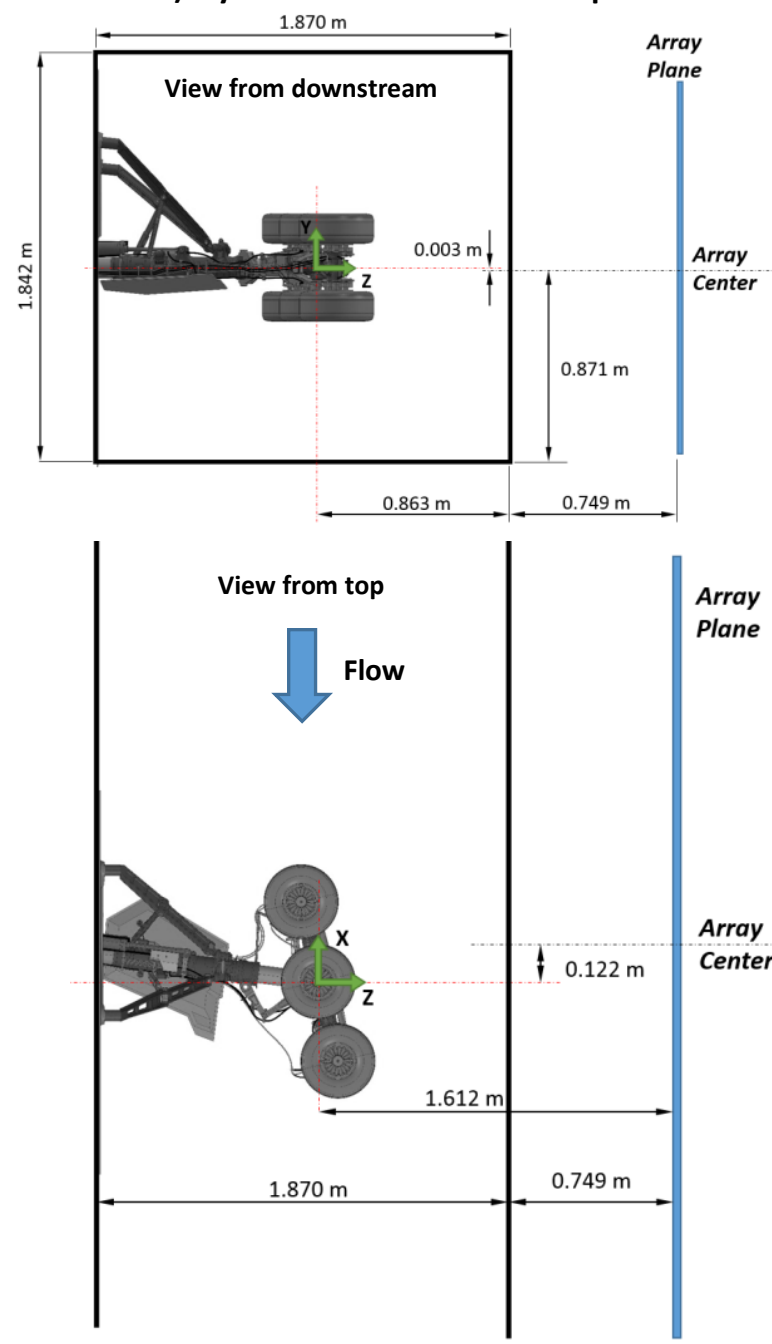

b) Sideline measurements setup
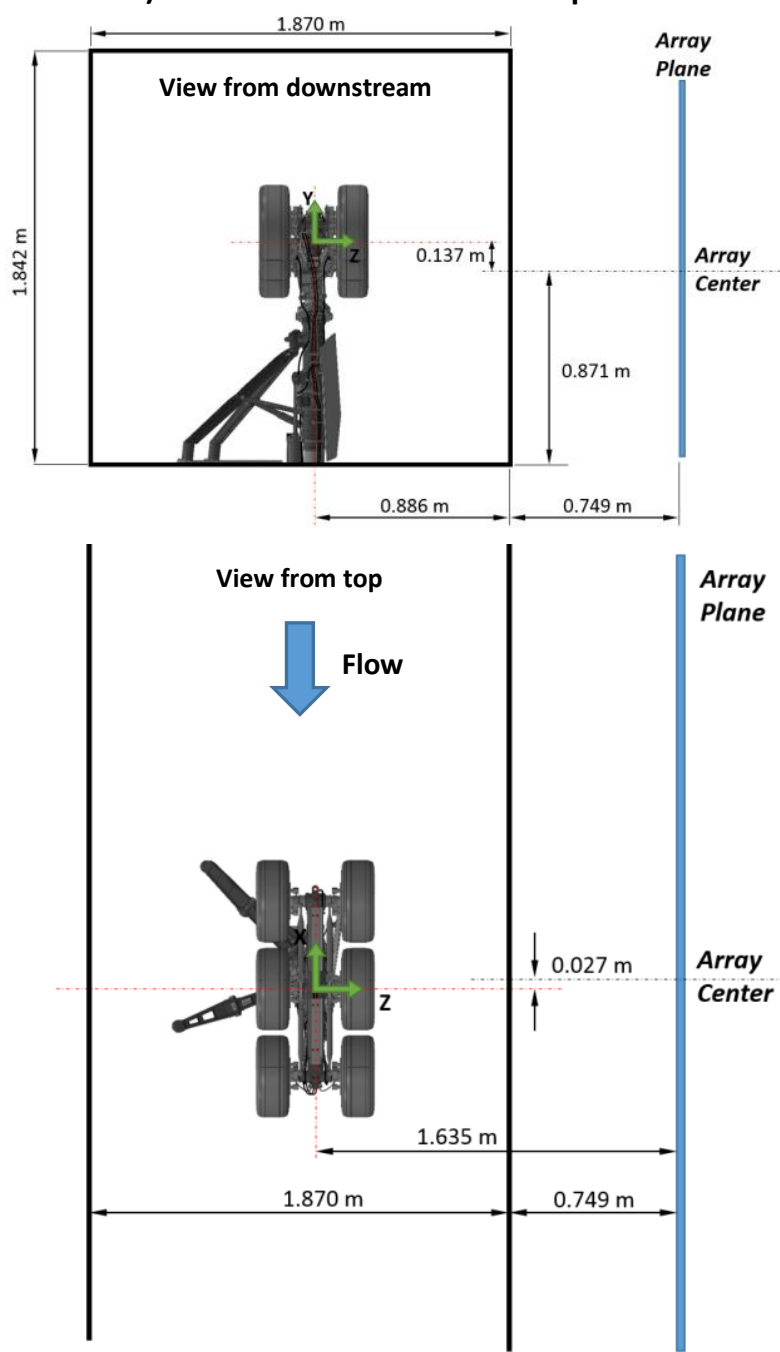

Figure 9. Schematics of array location relative to the model for a) flyover and b) sideline measurements. 


\section{E. Data Processing}

Beamforming was performed over a 3D grid surrounding the landing gear with a resolution of $1 \mathrm{~cm}$ in the plane parallel to the array, and $5 \mathrm{~cm}$ in the direction normal to it. This resulted in grids of 816,261 and 940,881 points for the flyover and sideline measurements, respectively. The beamforming algorithm accounts for diffraction at the boundary layer/Kevlar ${ }^{\circledR}$ window. Diagonal removal beamforming was used to reduce the impact of uncorrelated noise. Acoustic maps were obtained for narrowband frequencies between 250 and 22,500 Hz using a frequency resolution of $50 \mathrm{~Hz}$. Acoustic maps in $1 / 24^{\text {th }}, 1 / 12^{\text {th }}$, and $1 / 3^{\text {rd }}$ octave bands were obtained by adding the results from narrowband frequencies. Due to the frequency resolution of the narrowband results, some octave bands at low frequency had no energy (e.g. they are empty). All results in this work are presented in model-scale frequencies.

The array integrated spectra were computed for the entire 3D grid surrounding the landing gear following standard procedures $^{19}$ (i.e. normalizing by the point spread function for a source at the center of the $3 \mathrm{D}$ grid, accounting for diagonal removal, and applying a cutoff level to reduce the contribution from sidelobes). Even in this scenario, the integration results can be contaminated by sidelobes from other sources in the tunnel. This is particularly true for narrowband results. To further reduce the uncertainty on the integrated levels, the acoustic maps for all $1 / 12^{\text {th }}$ octave bands were visually inspected to ensure that actual sources were present and that the impact of sidelobes within the 5 $\mathrm{dB}$ integration cutoff was negligible. As expected, the frequency validity range for narrowband results was found to be lower than that for $1 / 12^{\text {th }}$, e.g., the narrowband maps are dominated by sidelobes starting at lower frequencies than those for $1 / 12^{\text {th }}$ maps. Samples of this scenario are shown later in this manuscript.

The spectra presented in this work (from average spectra, integrated spectra or maximum SPL in the acoustic maps) do not include the actual levels. However, these values were corrected to account for transmission losses at the $\operatorname{Kevlar}^{\circledR} /$ boundary layer and thus show the correct spectral shape. These corrections were estimated experimentally in a separate test (not yet published) using a methodology similar to the one published by Devenport et al. ${ }^{20}$

Data processing for the 251-element array was carried out with all the microphones in the array as well as seven subarrays with different microphone subsets: the four nested arrays shown before and combinations of them. The results presented in this work correspond to three sub-arrays obtained by combining two of the nested arrays in each sub-array, this is: Spirals 1-2 (S1-2), Spirals 2-3 (S2-3), and Spirals 3-4 (S3-4). Therefore, some of the microphones in each subarray are shared. The goal of beamforming with the sub-arrays was to obtain the noise levels as a function of directivity angle and to analyze differences in the characteristics of the acoustic maps. This type of array design using multiple
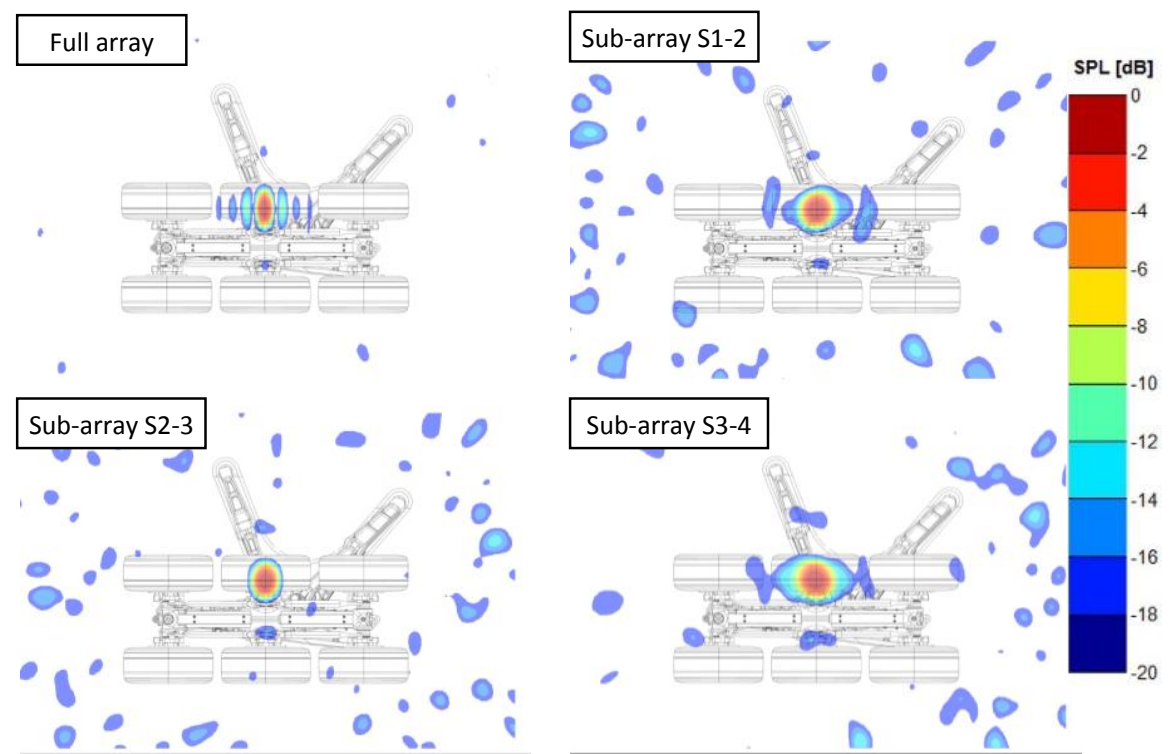

Figure 10. Point spread functions (PSF) for the full array and sub-arrays for the $1 / 12^{\text {th }}$ octave band with center frequency of $5,000 \mathrm{~Hz}$. Results for a plane at center of grid in the direction normal to the array. sub-arrays, referred as "pletharrays," was recently presented by Underbrink ${ }^{18}$ for different applications. Beamforming, integration, and generation of acoustic maps was performed using the commercial version of AVEC's Phased Array software. Sample array point spread functions (PSFs) for the whole array, as well as the sub-arrays mentioned above, are included in Figure 10 for the $1 / 12^{\text {th }}$ octave band with $5,000 \mathrm{~Hz}$ center frequency. The PSFs were plotted with contour levels $20 \mathrm{~dB}$ below the peak value in order to show the sidelobe structure. Since the sub-array S2-3 was widely used, additional PSFs for this sub-array are shown in Figure 11 for other frequencies. In all cases, the source is located at the center of the 3D scanning grid and only the plane at the source location is shown (see map over CAD model in Figure 11). As shown in Figure 12, the array signal-to-noise ratio (SNR, i.e., the level of the worst sidelobe relative to the peak level in the map) and the number of sidelobes is significantly reduced when obtaining the $1 / 12^{\text {th }}$ and $1 / 3^{\text {rd }}$ octave bands maps by adding the narrowband maps. Since the sidelobes are at slightly different locations for each 
narrowband frequency (within the lower and upper frequency of each octave band) while the main lobe (actual source) is always at the same location, the increase in the levels of the main lobe is larger than for the sidelobes, thus resulting in an increase in array SNR.

The integration was normalized by the PSF for a source at the center of the grid (since the entire grid was integrated to obtain the noise from the entire landing gear). However, the narrowband PSFs were not added to obtain the normalization value. Instead, the narrowband PSF for the center frequency of the corresponding octave band was used. This simplification did not impact the integration results because the integration cutoff level was set to 5 or $10 \mathrm{~dB}$ from the peak value and no sidelobes were within this threshold for the narrowband PSFs. Thus, small errors in the levels (with a standard deviation of about $0.1 \mathrm{~dB}$ for the baseline at $13^{\circ}$ ) are a result of slight changes in the main lobe size/shape. Given the large amounts of data and the size of the scanning grid, this conventional procedure was implemented to reduce computational time.
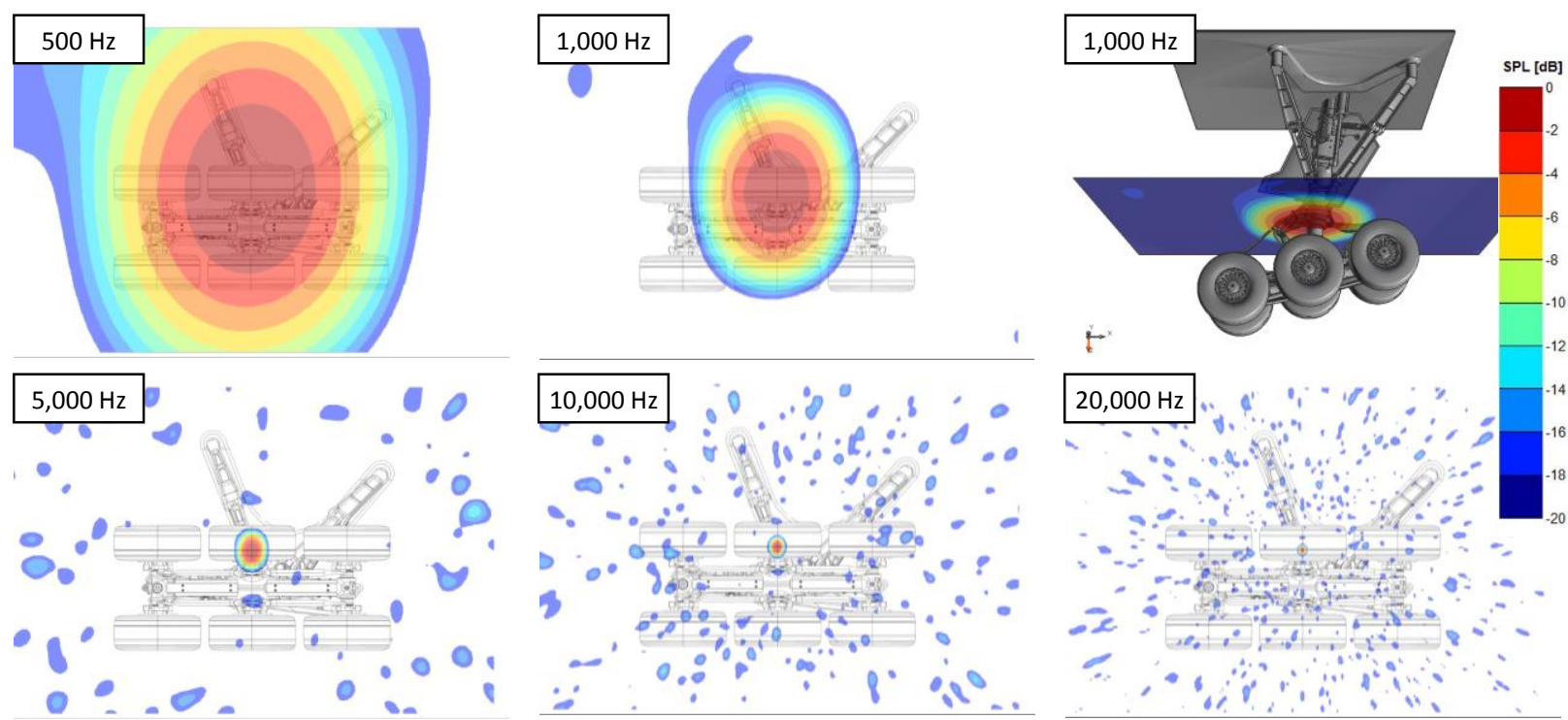

Figure 11. Point spread functions $\left(20 \mathrm{~dB}\right.$ contour levels) for sub-array $\mathrm{S} 2-3$ at different $1 / 12^{\text {th }}$ octave bands.
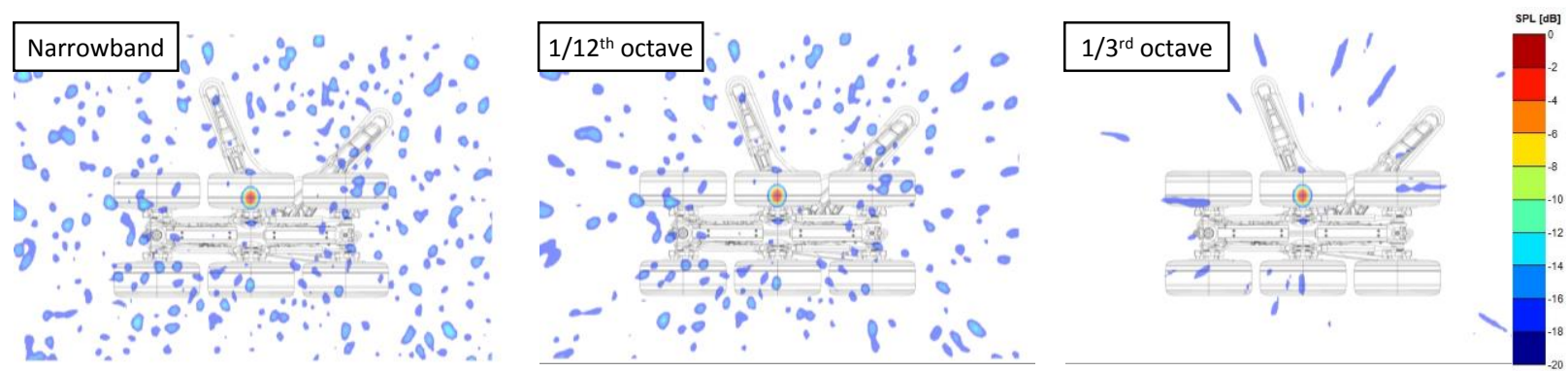

Figure 12. Point spread functions ( $20 \mathrm{~dB}$ contour levels) for sub-array $\mathrm{S} 2-3$. Results for narrowband, $1 / 12^{\text {th }}$ and $1 / 3^{\text {rd }}$ octave bands at a frequency of $10,000 \mathrm{~Hz}$. The octave band maps were obtained by adding the narrowband maps within the band (using the same frequency resolution than the analysis, e.g., $50 \mathrm{~Hz}$ ).

\section{Analysis of Results}

As mentioned in section I, the main goals of the test were to generate a database of acoustic data for validation of simulations and to better quantify the noise reduction capabilities of the toboggan fairing. The latter is an effort to reconcile the differences seen from previous wind tunnel tests and flight test data from the QTD2 campaign. ${ }^{6}$ The results presented here include sample acoustic maps obtained with the new instrumentation and a comparison of the acoustic signature of the toboggan faring to the baseline configuration.

All the results in this section are presented in model-scale frequencies. Unless otherwise noted, the cutoff level for a contour plots is $10 \mathrm{~dB}$ from the peak value in the entire 3D grid. If a different cutoff is used for the contour plot, an orange border was added to the map and the new cutoff level is indicated. In most cases, if two configurations are 
being compared, the maximum value in both cases was set to the same value to aid in the comparison. In some cases, the maps for a quieter configuration would not show any sources using the same maximum level than in the baseline configuration. In these particular cases, a red border was added to the map and the relative level of the peak value is included for reference.

Unless noted, the sub-array comprised of spirals 2 and 3, and referred as Spirals 2-3 or S2-3, was used as reference for most of the analysis. Although a 3D grid around the landing gear was beamformed, most figures only show a plane parallel to the array where the maximum level was found or an interesting feature was observed. Note that due to poor array resolution in the direction normal to it, a source from a different plane might show up as a source with lower levels at a different plane. Sample "3D maps" will be presented later in this section (see Figure 18 and Figure 19).

Sample acoustic maps for the baseline and the toboggan configuration (for a truck angle of $13^{\circ}$ ) are shown in Figure 13 and Figure 14 for different $1 / 12^{\text {th }}$ octave band frequencies ranging between 500 and 20,000 $\mathrm{Hz}$. The corresponding center frequency of each band is indicated on the top left corner of each row of maps.

In the example for $500 \mathrm{~Hz}$, both configurations show virtually the same maximum level, and thus the toboggan shows no impact at this frequency. However, the maximum level is in a slightly different $\mathrm{Z}$ plane. This was also observed for other frequencies around $500 \mathrm{~Hz}$. As frequency increases the noise reduction effect of the toboggan is more noticeable, even for sources around the main strut and side braces. Around $1,000 \mathrm{~Hz}$, the dominant source for the baseline configuration still appears to be around the main strut and braces.

However, for higher frequencies, the dominant sources observed with
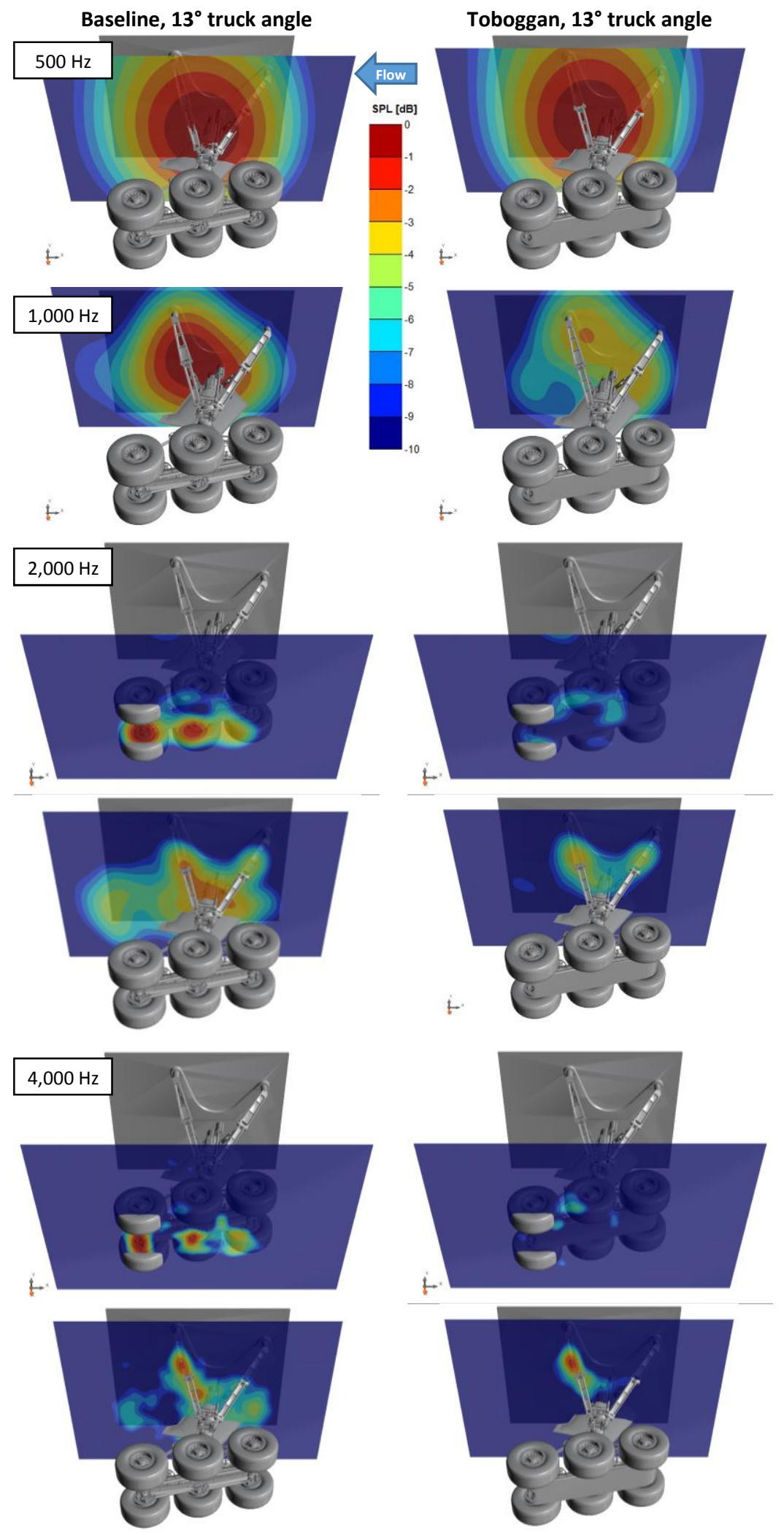

Figure 13. Comparison of $1 / 12^{\text {th }}$ octave band acoustic maps (S2-3) for baseline and toboggan configurations. $M=0.17,13^{\circ}$ truck angle. 
the sub-array S2-3 for the baseline configuration at $13^{\circ}$ are always located around the truck. For the toboggan configuration at $2,000 \mathrm{~Hz}$, all major sources on the truck "disappear." The levels of the remaining sources at this particular plane are now $\sim 6 \mathrm{~dB}$ below those for the baseline configuration. These lobes result from the actual sources at this frequency (the braces) being in a different plane, as shown in the lower row of results for $2,000 \mathrm{~Hz}$. When compared to the baseline, some noise reduction is also observed around the main strut/braces. Again, keep in mind that some lobes in this plane correspond to sources in the truck. Similar results are observed for $4,000 \mathrm{~Hz}$. In this example only the aft brace appears as a major noise source when the toboggan is installed.

As shown in Figure 14, for frequencies around $6,000 \mathrm{~Hz}$ and above, the baseline configuration only shows dominant sources around the truck area (e.g. aft, center, and forward axles/brakes). The toboggan fairing very efficiently reduces the noise levels at high frequencies. For instance, at $6,000 \mathrm{~Hz}$ the toboggan configuration shows no sources around the truck area within $10 \mathrm{~dB}$ of the baseline levels. In fact, the dominant source is now around the aft brace and its maximum level is about $9 \mathrm{~dB}$ below the maximum level for the baseline. Some contamination of the acoustic maps (from sidelobes due to the relatively low levels of this configuration) is starting to be visible downstream of the landing gear. Similar results are observed for $12,500 \mathrm{~Hz}$, with clear sources in the forward and aft axle/brakes region in the baseline configuration. The toboggan configuration again shows a dominant source at the aft brace (and others around the main strut and forward brace), with the maximum level in the map being $7.7 \mathrm{~dB}$ below the maximum level observed for the baseline. Note that the number of sidelobes is now significantly increased, to the point that the integrated levels at this frequency would be inaccurate for the toboggan configuration. For the sample maps at higher frequencies, the baseline again shows clear sources in the aft region of the truck. However, contamination in the maps for the toboggan configuration is so severe that it is hard to unequivocally identify the actual noise sources and much less feasible to obtain an accurate estimate of the integrated levels. If the results
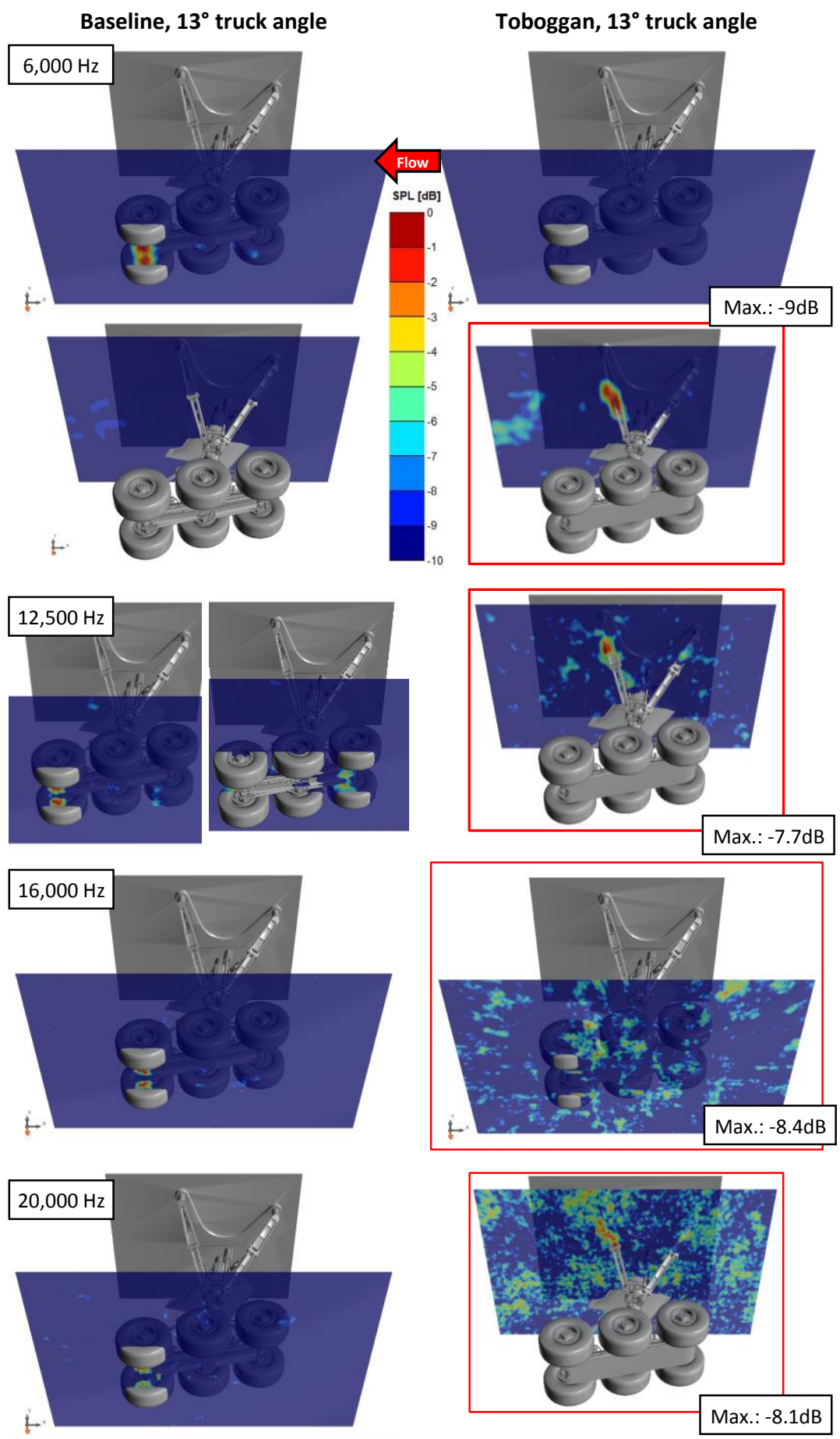

Figure 14. Comparison of $1 / 12^{\text {th }}$ octave bands acoustic maps (S2-3) for baseline and toboggan configurations. $\mathrm{M}=0.17,13^{\circ}$ truck angle. 
for $16,000 \mathrm{~Hz}$ are examined more closely, noise sources are observed between the wheels and the toboggan. Similar sources in these locations were observed for the $0^{\circ}$ truck angle, where they become more evident and easy to identify.

Results for the baseline and toboggan configurations at $0^{\circ}$ truck angle are shown in Figure 15 through Figure 17 for the same set of $1 / 12^{\text {th }}$ octave band frequencies presented before. In these cases, the characteristics of the acoustic maps change significantly. Unlike the $13^{\circ}$ truck angle case, the toboggan configuration is now louder than the baseline at frequencies below $~ 900$ $\mathrm{Hz}$. In the example for $500 \mathrm{~Hz}$ in Figure 15, the dominant source for the toboggan configuration is in a slightly different location than for the baseline and its level is about 4 dB louder. Similar results were also observed from the sideline measurements, suggesting that the presence of the toboggan might be accelerating the flow in the upper section of the truck. This change in the location of the sources is also seen for the $1,000 \mathrm{~Hz}$ maps. However, the peak level is about $1.4 \mathrm{~dB}$ lower for the toboggan (while due to the size/shape, the integrated levels show no change at this frequency).

For $2,000 \mathrm{~Hz}$, the dominant sources for the baseline is in the truck. However, unlike the $13^{\circ}$ truck angle case, the dominant source appears in the forward region. Once the toboggan is installed, the dominant sources shift to locations between the toboggan and the wheels, most likely caused by flow acceleration around other components in the upper part of the truck. Sources in the upper section of the main strut and braces are also visible (not shown here), with levels similar to those for the baseline configuration.

Similar trends are observed for 4,000 Hz. However, the noise from the upper truck section is now less dominant for the toboggan configuration. At this frequency, the maps for the baseline and toboggan show similar sources in the aft side brace.
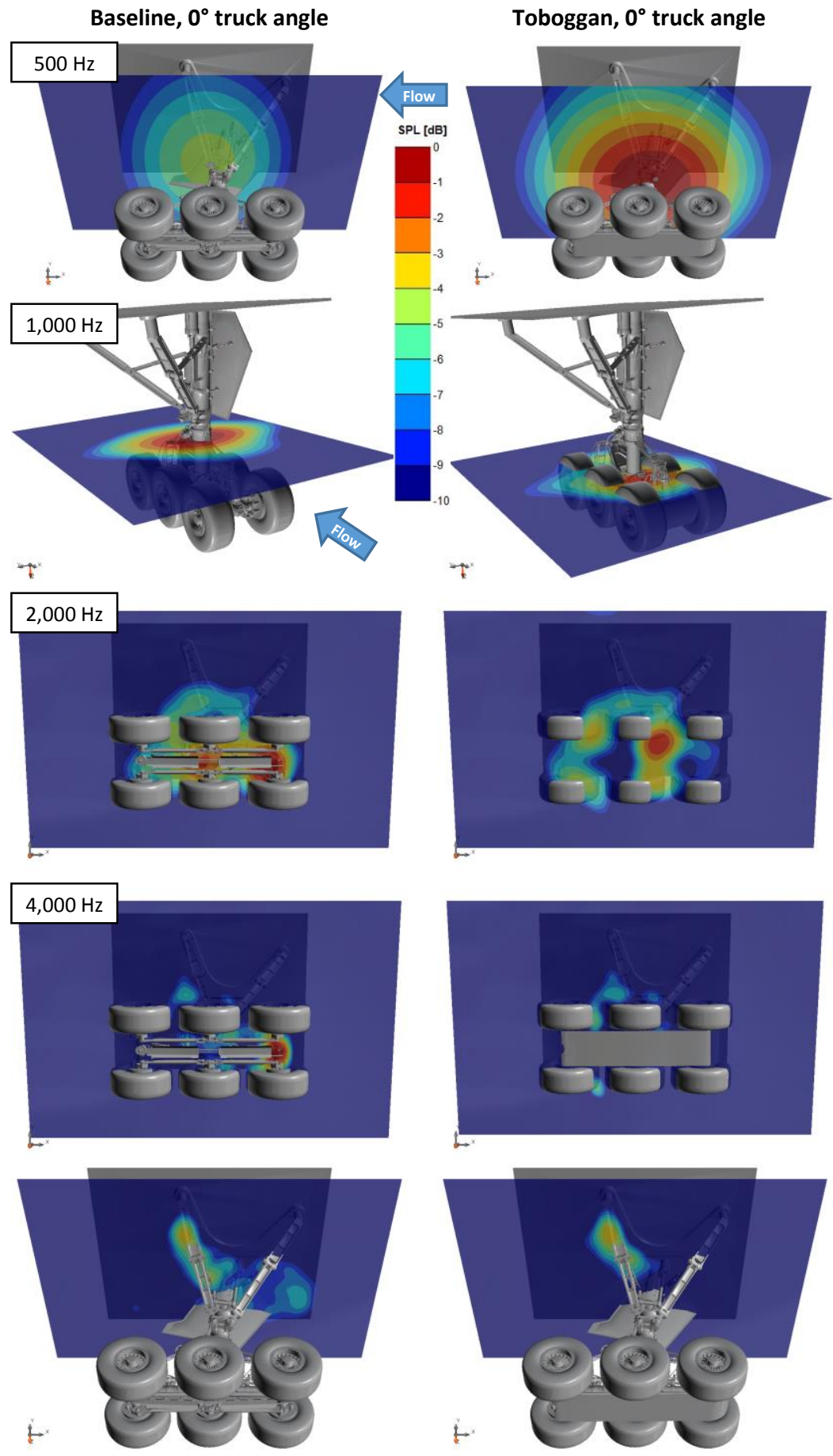

Figure 15. Comparison of $1 / 12^{\text {th }}$ octave bands acoustic maps (S2-3) for

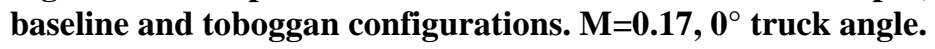

The baseline configuration also shows and additional source (with lower levels) at the junction of the main strut and tunnel floor. This source becomes more evident if the acoustic map for a different plane is analyzed. 
Figure 16 shows comparisons for $6,000,8,000$ and $12,500 \mathrm{~Hz}$. At all of these frequencies the baseline maps show the dominant sources in the forward section of the truck and installing the toboggan eliminates them. Around $6,000 \mathrm{~Hz}$, the aft brace becomes the dominant source (with levels similar to those seen for the aft brace in the baseline configuration), and two new sources appear in the forward section of the aft wheel. Other sources with slightly lower levels are also visible between the toboggan and the center and aft wheels (not shown here).

For frequencies around 8,000 $\mathrm{Hz}$, both configurations show slightly more sidelobes than for surrounding frequencies. For the baseline this behavior is likely due to the large number of sources seen in the truck, while in the case of the toboggan configuration it is most likely related to sidelobes of other sources in the wind tunnel. Interestingly, the faired configuration shows a noise source in the toboggan section facing the flow. Further inspection revealed that this source coincides with the location of a bolt used to attach the flexible material to the SLS structure of the toboggan. Close to this frequency, a line of sources was also observed around the upper lip of the toboggan in the region facing the flow (see Figure 6).

At $12,500 \mathrm{~Hz}$, somewhat unexpected results were observed. For the baseline case, the dominant source was pinpointed to a protruding component in the forward axle near the tow hook and the two brackets around the hydraulic valves (under the rock guards). In the toboggan configuration, noise is clearly radiated along the edge of the toboggan. Note that the dominant source in the toboggan is located in the upper section of the aft brace, not shown here. Several sidelobes are present at this frequency also. However, using an integration cutoff of $5 \mathrm{~dB}$, their contribution would not significantly impact the levels of the actual sources.

Figure 17 compares the acoustic maps at 16,000 and 20,000 Hz. The dominant sources for the baseline are in the locations described for the map at $12,500 \mathrm{~Hz}$. For the toboggan, the dominant source is at the center of the aft brace, with a peak level over $8 \mathrm{~dB}$ lower than the peak level for the baseline. The presence of several sidelobes prevents a clear identification of sources around the truck in these maps. Closer inspection reveals sources between the (forward and center) wheels and the toboggan, similar to the $12,500 \mathrm{~Hz}$ case. At $20,000 \mathrm{~Hz}$, the baseline shows a dominant source around the bolts used to attach the forward rock guard to the hydraulic valves. The maximum levels in the map for the toboggan configuration are over $10 \mathrm{~dB}$ below the baseline levels. The map with a smaller cutoff facilitates identification of a source around the aft brace. However, the level and number of sidelobes present make it unlikely that an integrated level could be accurately determined, even with a cutoff of $5 \mathrm{~dB}$. 

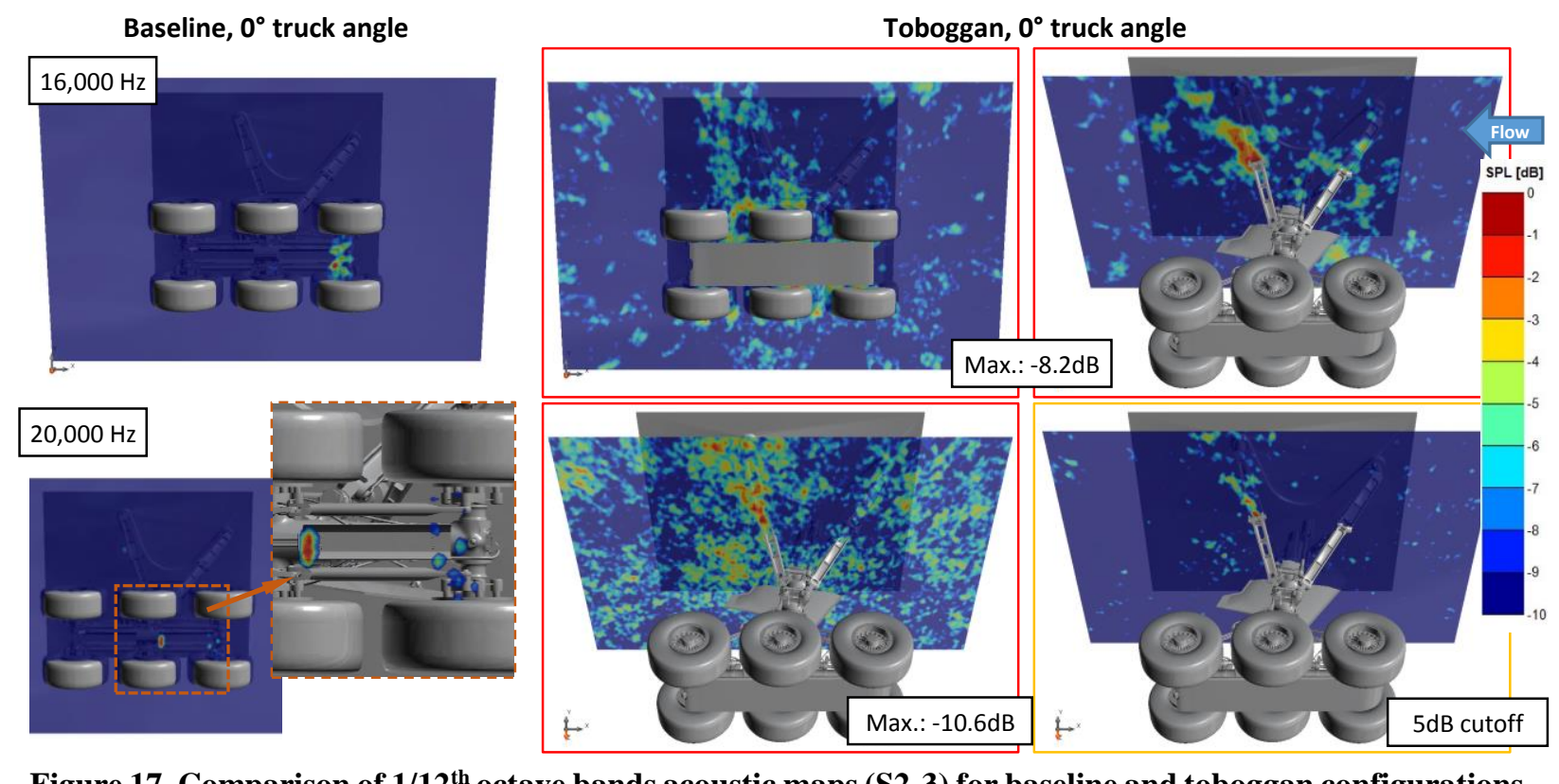
Figure 17. Comparison of $1 / 12^{\text {th }}$ octave bands acoustic maps (S2-3) for baseline and toboggan configurations.
$M=0.17,0^{\circ}$ truck angle.

After analyzing the acoustic maps to determine the major noise sources, the next step was to quantify the noise reduction for the toboggan configuration. If the landing gear were the only acoustic source in the wind tunnel, this could be accomplished by simply looking at the spectra of individual microphones. However, as will be shown later, the wind tunnel background noise levels are relatively high and thus the noise produced by the landing gear cannot be quantified in this manner. The beamforming results allow separation of landing gear noise from other sources in the tunnel. This noise decomposition is typically accomplished by integrating the acoustic maps to obtain the levels at each frequency. Ideally, the integration would be carried out with the largest possible cutoff level to include the contribution from all sources. However, as was shown in the examples before, depending on the configuration, the acoustic maps at high frequencies may be contaminated by sidelobes from sources not on the landing gear or from uncorrelated noise. The fact that the PSFs do not show any sidelobes within $10 \mathrm{~dB}$ of the peak value means that, once the toboggan is installed, the levels at high frequencies are reduced so drastically that the background noise in the wind tunnel becomes dominant. Hence, the sources observed in the acoustic maps mostly result from contamination or from uncorrelated noise. Since sidelobe levels in a PSF are for a single source at the center of the grid, the presence of multiple sources or sources far from the center of the grid would likely introduce sidelobes within the integration region and render the integration of the acoustic maps inaccurate. By normalizing the integration by the PSF, the sidelobes due to sources on the landing gear are already accounted for. However, all other spurious sources artificially increase the integrated levels. Sample "3D" acoustic maps (maps showing the contour levels for each plane normal to the array, shown as gray layers) for the baseline and toboggan configurations at a truck angle of $13^{\circ}$ are shown in Figure 18 with a cutoff of $5 \mathrm{~dB}$ and in Figure 19 for a cutoff of $10 \mathrm{~dB}$. Figure 18 clearly shows that no extraneous sidelobes are present for the baseline results. However, around $10 \mathrm{kHz}$ the toboggan configuration already shows multiple sidelobes (some of which might still be due to the presence of multiple sources on the landing gear) that could affect the integrated level. For $18 \mathrm{kHz}$, the number and level of the sidelobes clearly dominate the maps and hence the integrated levels are not expected to represent the actual level of the sources on the landing gear. This would result in a clear under estimation of the noise reduction obtained with the toboggan fairing. Figure 19 shows that, with the integration cutoff set to $10 \mathrm{~dB}$, the "accurate" frequency range would be further reduced for the toboggan configuration. 


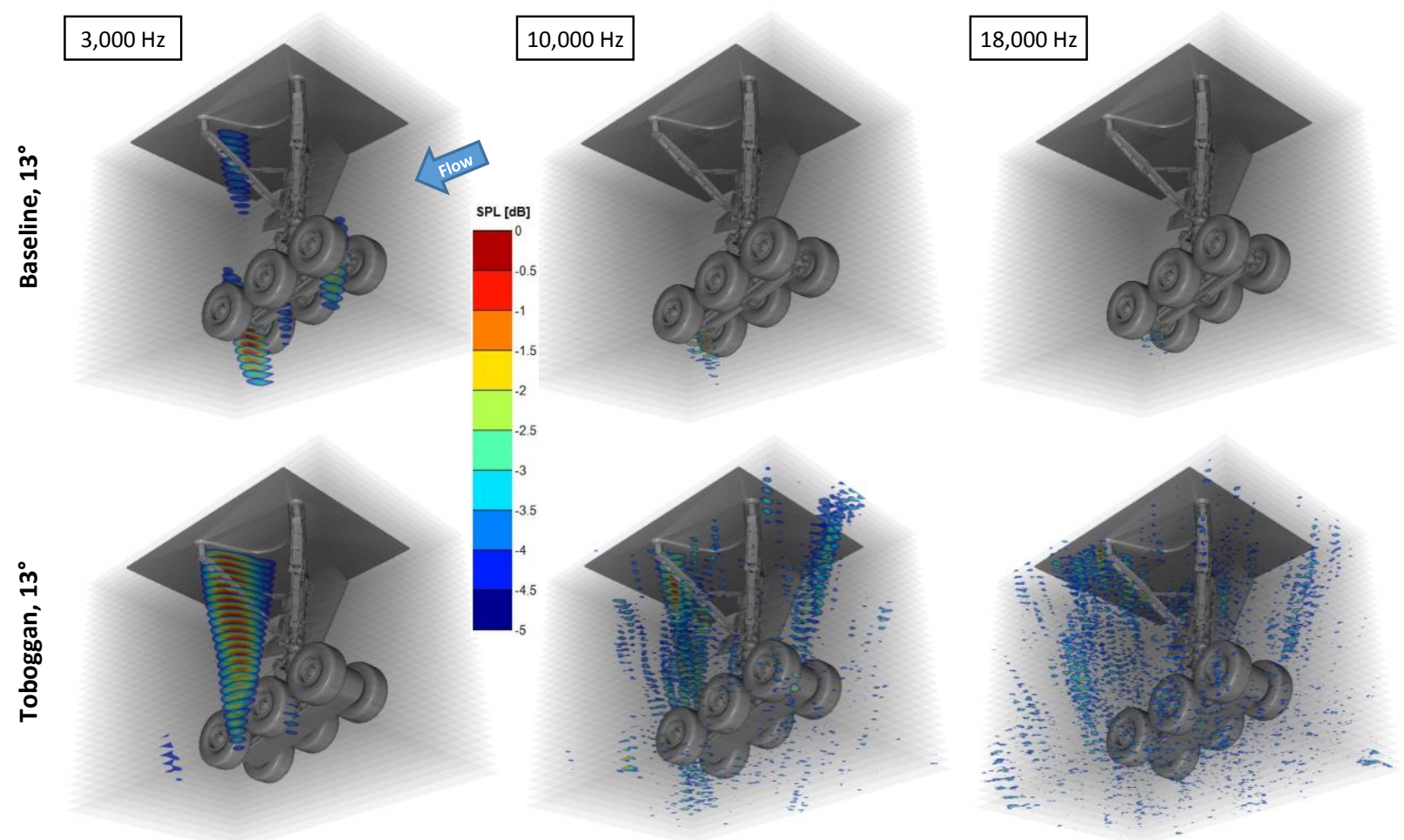

Figure 18. Sample 1/12 ${ }^{\text {th }}$ octave bands "3D" acoustic maps for sub-array S2-3 showing levels being integrated when a cutoff of $5 \mathrm{~dB}$ is used. Contour levels in each figure set to maximum in 3D grid.

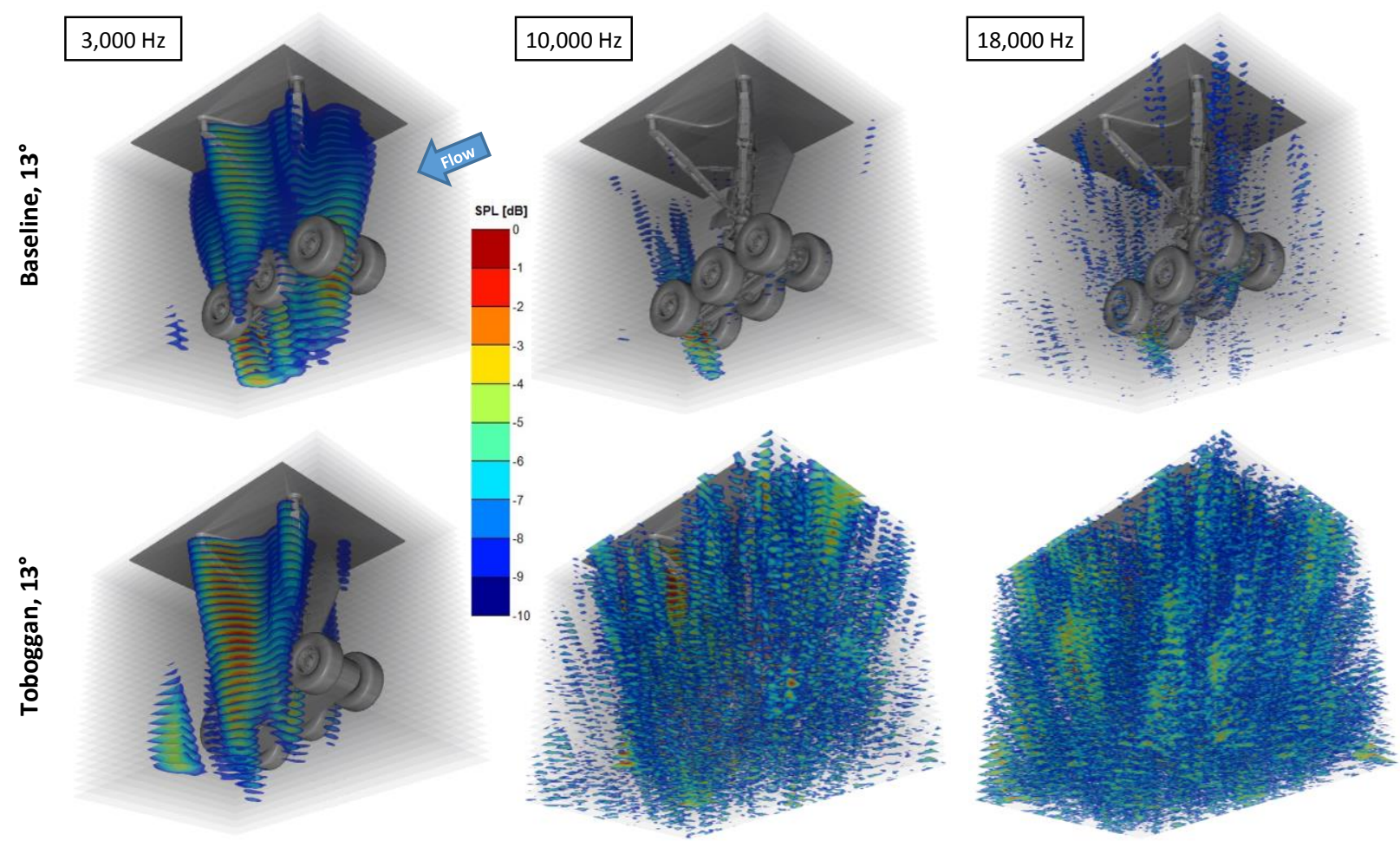

Figure 19. Sample 1/12 ${ }^{\text {th }}$ octave bands "3D" acoustic maps for sub-array S2-3 showing levels being integrated when a cutoff of $10 \mathrm{~dB}$ is used. Contour levels in each figure set to maximum in 3D grid. 
To avoid misinterpretation of the integrated levels due to the impact of these sidelobes, the $1 / 12^{\text {th }}$ octave band acoustic maps for all configurations were visually inspected using a $5 \mathrm{~dB}$ cutoff to determine the valid frequency range. In some cases, intermediate frequencies were slightly contaminated but they were kept in an effort to maximize the frequency range (i.e., if a map was slightly contaminated but subsequent frequencies were not, the highest frequency was used). An example of this analysis is shown for the baseline and toboggan configurations in Figure 20 for the flyover measurements. In this case, the integrated spectra values for valid frequencies are represented using solid lines, while values for which contamination was observed are represented using dashed lines. As can be seen for the sideline measurements in Figure 21, the impact of the toboggan on the maximum levels (and hence the sidelobes) on the maps was not as significant, and therefore the valid frequency range for the toboggan configuration in this orientation is wider than that in the flyover direction.

Since the PSF used to normalize the integrated levels at each frequency is located at the center of the 3D grid and the distance between any grid point and the center of the grid is not negligible, errors for sources that are not close to the center of the grid would also be introduced. That is, sources further away from the array (with a larger main lobe) would be overestimated and sources closer to the array (with a smaller main lobe) would be underestimated. Integration of simulated PSFs in off-center locations (e.g., aft brakes or center of aft brace) show that these variations can be about $2 \mathrm{~dB}$ below $10,000 \mathrm{~Hz}$, and up to $3 \mathrm{~dB}$ at higher frequencies. On the other hand, attempting to integrate individual components (regions in space) and adding their spectral contribution would suffer from inclusion of spurious sources.

Another option to quantify the noise consists of looking at the maximum levels at each frequency to obtain a spectrum. This can be seen as being equivalent to the integrated spectra obtained with a cutoff value of zero. The advantage of this approach is that the levels are not contaminated by sidelobes (unless the peak level in the map does not correspond to a source on the model). The main disadvantage is that it does not account for the number of sources, their shape, or size (i.e., for distributed sources). As a result, noticeable differences emerge when comparing the levels to the average or integrated spectra, as will be shown below. Despite this, the approach produces useful results that can be used to quickly show trends, in particular for frequencies in which the integrated spectra was deemed inaccurate.

Figure 22 shows the flyover average spectra, integrated spectra (using cutoff values of 5 and $10 \mathrm{~dB}$ from the peak value) and the maximum SPL in the maps for the baseline and toboggan configurations at $13^{\circ}$ truck angle. Under simple, ideal conditions (single source in anechoic environment), all these levels would be the same. However, during normal testing, the integrated spectra levels would fall between the values of the average spectra (upper bound) and the maximum levels (lower bound). In the presence of multiple sources with different levels at a given frequency, the integration cutoff determines which sources' contributions are accounted for. Therefore, as the integration cutoff is increased, the levels should better resemble the average spectra. If relatively high background noise levels are present, which is typical in a wind tunnel environment, even an "ideal" integrated spectra would not reach average spectra levels. Also, as shown in Figure 22, the integrated spectra exceeded the average spectra levels at high frequencies, where the contribution of the sidelobes and extraneous sources is significant. This effect becomes more prominent as the integration cutoff is increased, as evidenced by the fact that the "cross-over" between average and integrated spectra occurs at lower frequencies as the cutoff level is increased. Note also that in this case, the contamination actually starts at frequencies below the cross-over value.

A similar comparison using sideline measurements is shown in Figure 23. Analysis of the $1 / 12^{\text {th }}$ octave maps (with a $5 \mathrm{~dB}$ cutoff) shown in Figure 21 identified clear sources at the model and no contamination for the baseline and toboggan configurations. Little to no contamination was observed for a cutoff of $10 \mathrm{~dB}$. This behavior is consistent with the fact that the integrated spectra do not show levels higher than the average spectra. Note that for some frequencies (see levels around 1,200 Hz in Figure 23) the maximum SPL shows an "increase in noise" for the toboggan configuration while the integrated spectra depicts a reduction in noise. This fact further reinforces that, in general, 1) maximum SPL alone should not be used as a criterion to quantify noise reduction; and 2) "noise reduction from maximum levels" (i.e., the difference in maximum map levels between two configurations) cannot be labeled as being an upper or lower bound on the expected noise reduction, regardless of the fact that the maximum levels were the lower bound for the integrated spectra (for frequencies in which at least one landing gear noise source is visible).

On the other hand, since the average spectra is the upper bound of the integrated levels, the difference in average spectra levels between two configurations (with the corresponding sign for reduction or increase) is also the lower bound of the change in integrated levels (assuming incoherent sources and that the wind tunnel background levels do not change significantly between configurations). However, for differences approaching zero, whether the average spectra levels are a lower bound for noise reduction or an upper bound for an increase in noise cannot be determined because of variability in the measurements. Also note that the (single microphone) SNR between the background noise and the sources of interest would determine the accuracy of this bound, e.g., high background levels would result 
in lower noise reduction levels for the source of interest (again, assuming background levels do not change significantly between configurations, which is the case in this work).

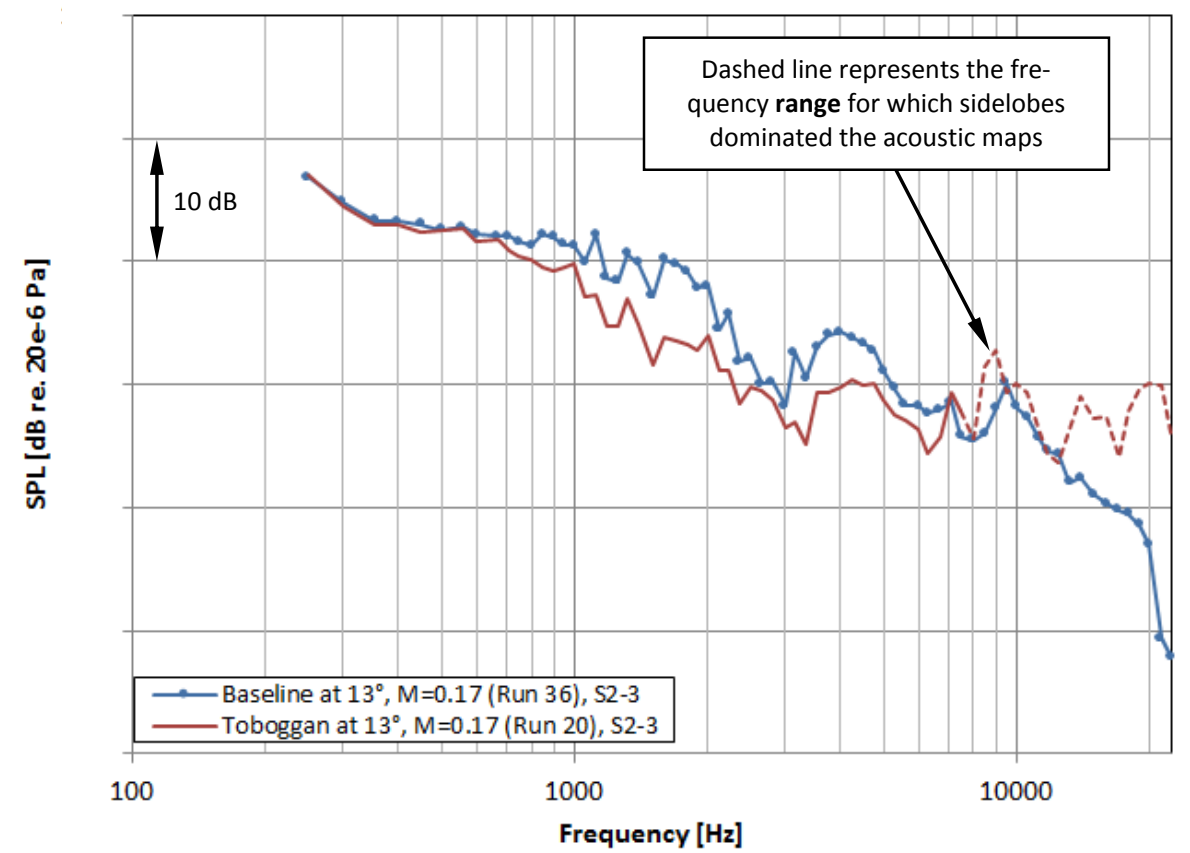

Figure 20. Comparison of flyover integrated spectra ( $5 \mathrm{~dB}$ cutoff) for baseline and toboggan configuration at $13^{\circ}$ truck angle. Results in $1 / 12^{\text {th }}$ octave bands from sub-array S2-3.

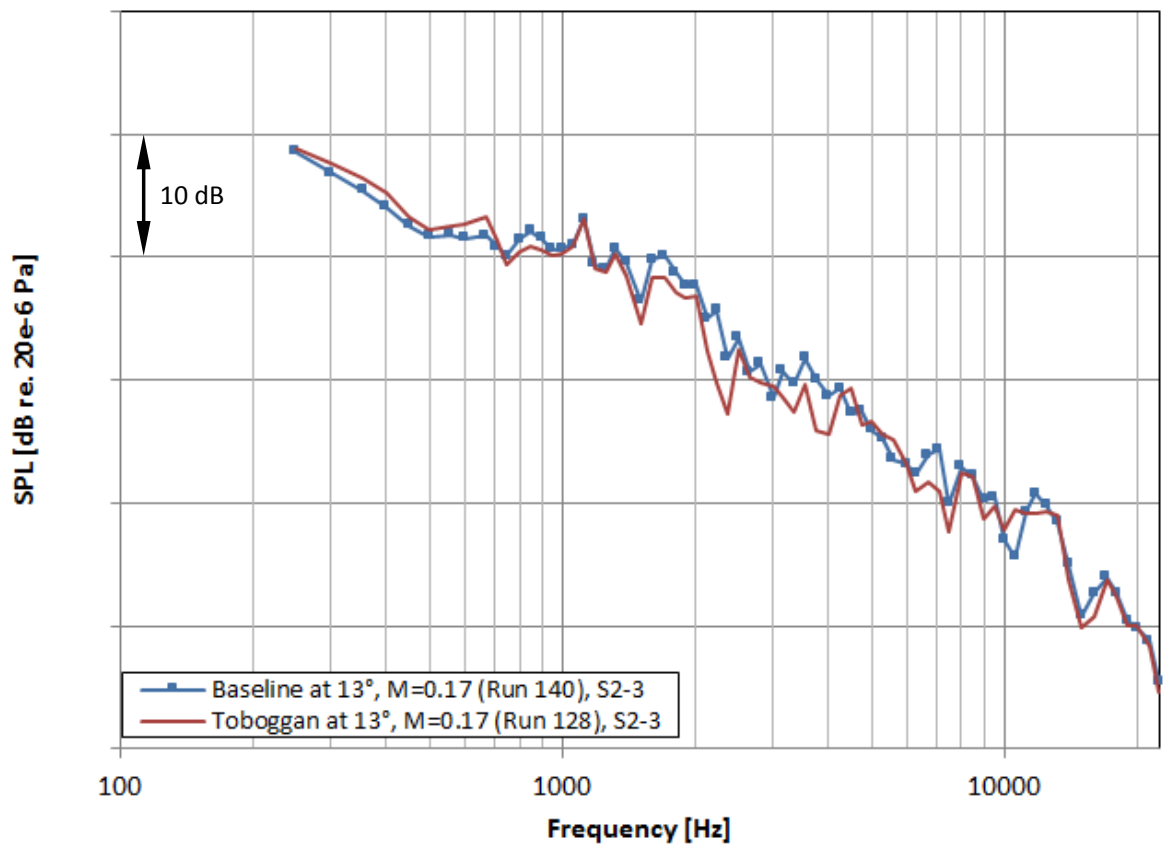

Figure 21. Comparison of sideline integrated spectra ( $5 \mathrm{~dB}$ cutoff) for baseline and toboggan configuration at $13^{\circ}$ truck angle. Results in $1 / 12^{\text {th }}$ octave bands from sub-array $\mathrm{S2-3}$. 


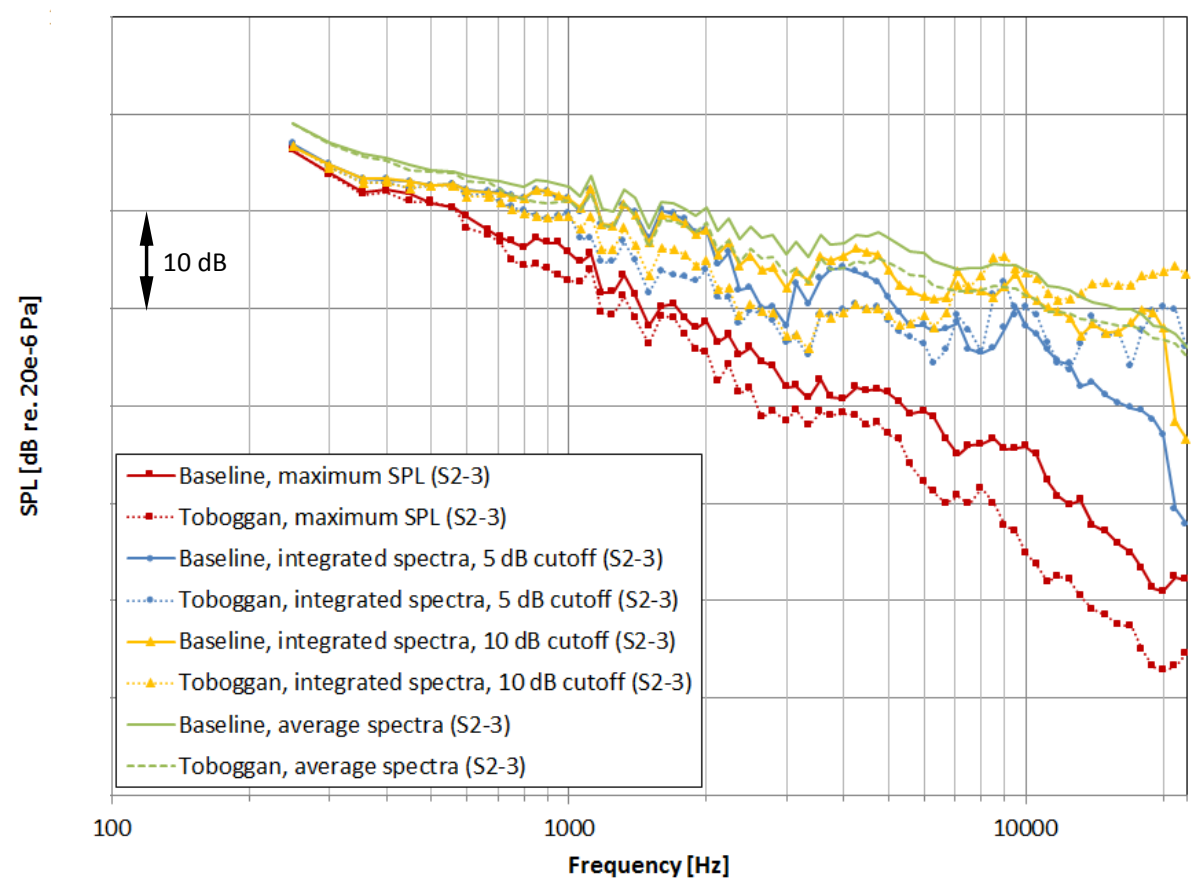

Figure 22. Comparison of flyover average spectra, integrated spectra, and maximum SPL in the maps for baseline and toboggan configurations at $13^{\circ}$ truck angle. Results in $1 / 12^{\text {th }}$ octave bands from sub-array S2-3.

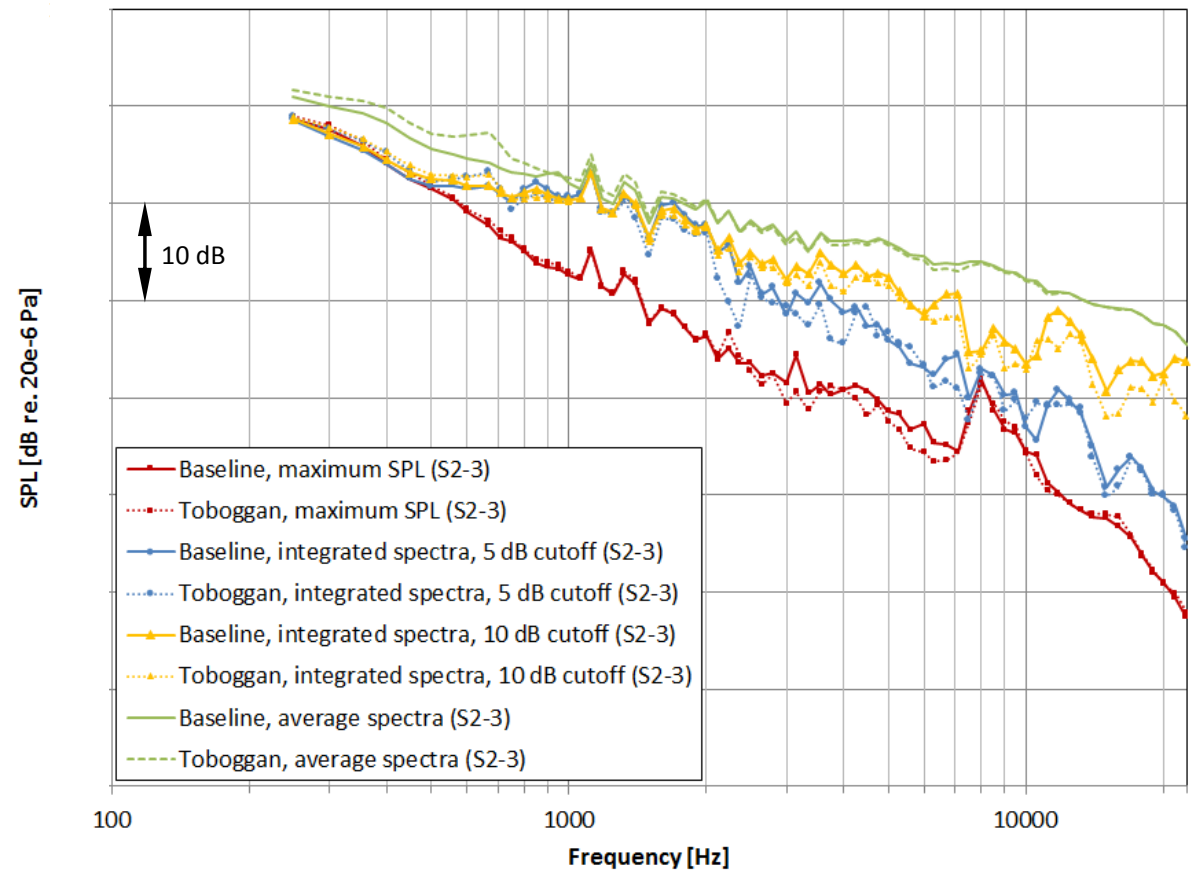

Figure 23. Comparison of sideline average spectra, integrated spectra, and maximum SPL in the maps for baseline and toboggan configurations at $13^{\circ}$ truck angle. Results in $1 / 12^{\text {th }}$ octave bands from sub-array S2-3.

To further illustrate these differences, the magnitude of noise reduction (between baseline and toboggan configurations) obtained from average spectra, integrated spectra and maximum levels are presented. As can be seen in Figure 24 for flyover measurements at $13^{\circ}$ truck angle, the values from the integrated levels indicate an increase in noise for

American Institute of Aeronautics and Astronautics 
the toboggan configuration above $\sim 7,000 \mathrm{~Hz}$. In contrast, the average spectra show noise reduction for the same frequencies. This further indicates that, as shown before, the toboggan integrated levels are contaminated in that frequency range. In this particular case, integration with a $10 \mathrm{~dB}$ cutoff clearly indicates that the values could be invalid above $\sim 7,000 \mathrm{~Hz}$ (Figure 22). However, the integration with a $5 \mathrm{~dB}$ cutoff was only greater than the average spectra for a few values above $\sim 9,000 \mathrm{~Hz}$, and thus not providing an indication of invalid results. The noise reduction plot in Figure 24 suggests that the levels above $7,000 \mathrm{~Hz}$ are not accurate, which is consistent with the visual analysis of the acoustic maps. Therefore, comparison with the noise reduction from the average spectra results can provide further insight from the previous analysis (i.e. simply comparing the cross-over frequency and neglecting the frequencies for which the integrated spectra are higher than the average spectra). Unfortunately, how much this lower bound for the noise reduction approaches the actual noise reduction in the model is also affected by the relative levels between the test subject and the facility, and could,therefore, be underestimated.

In conclusion, Figure 24 indicates that the toboggan provides noise reduction for most frequencies. The noise reduction is between 2.5 and $7 \mathrm{~dB}$ for frequencies within the range 800 to $7,000 \mathrm{~Hz}$ (based on the integration with a $10 \mathrm{~dB}$ cutoff). Using the average spectra values, the expected noise reduction would be at least 2 to $3 \mathrm{~dB}$ between 7,000 and $18,000 \mathrm{~Hz}$. The noise reduction for frequencies below $800 \mathrm{~Hz}$ is less than $2 \mathrm{~dB}$. Figure 24 shows that the noise reduction from integrated levels with a cutoff of $5 \mathrm{~dB}$ drops below the values from the average spectra and that the ones with a $10 \mathrm{~dB}$ cutoff are close to those with a $5 \mathrm{~dB}$ cutoff. To highlight what is happening around these frequencies, Figure 25 shows 3D acoustic maps for $2,650 \mathrm{~Hz}$ with contour levels 3,5 and $10 \mathrm{~dB}$ below the maximum in the maps (i.e., to illustrate what would be integrated using such cutoff levels). As can be seen, the "volume" being integrated for the toboggan configuration is larger than the one for the baseline. Therefore, although the peak level for the loudest source in the toboggan configuration is $5 \mathrm{~dB}$ below the peak value for the baseline configuration, the fact that there are more sources within the integration cutoff value results in larger integrated levels for the toboggan configuration, and therefore an underestimation of the noise reduction. As the integration cutoff increases, these differences become smaller. An alternative to overcome this issue would be to integrate using the maximum peak value among the configurations being compared. However, besides making the processing and analysis more complex, this approach would not provide an accurate representation of the noise reduction because the contribution of some sources would be neglected. For instance, in the example of Figure 25, integrating with a $5 \mathrm{~dB}$ cutoff from the peak value in the baseline case would render a very small value for the toboggan configuration (no values exist within $5 \mathrm{~dB}$ of the baseline levels) and thus the noise reduction would be significantly over predicted.

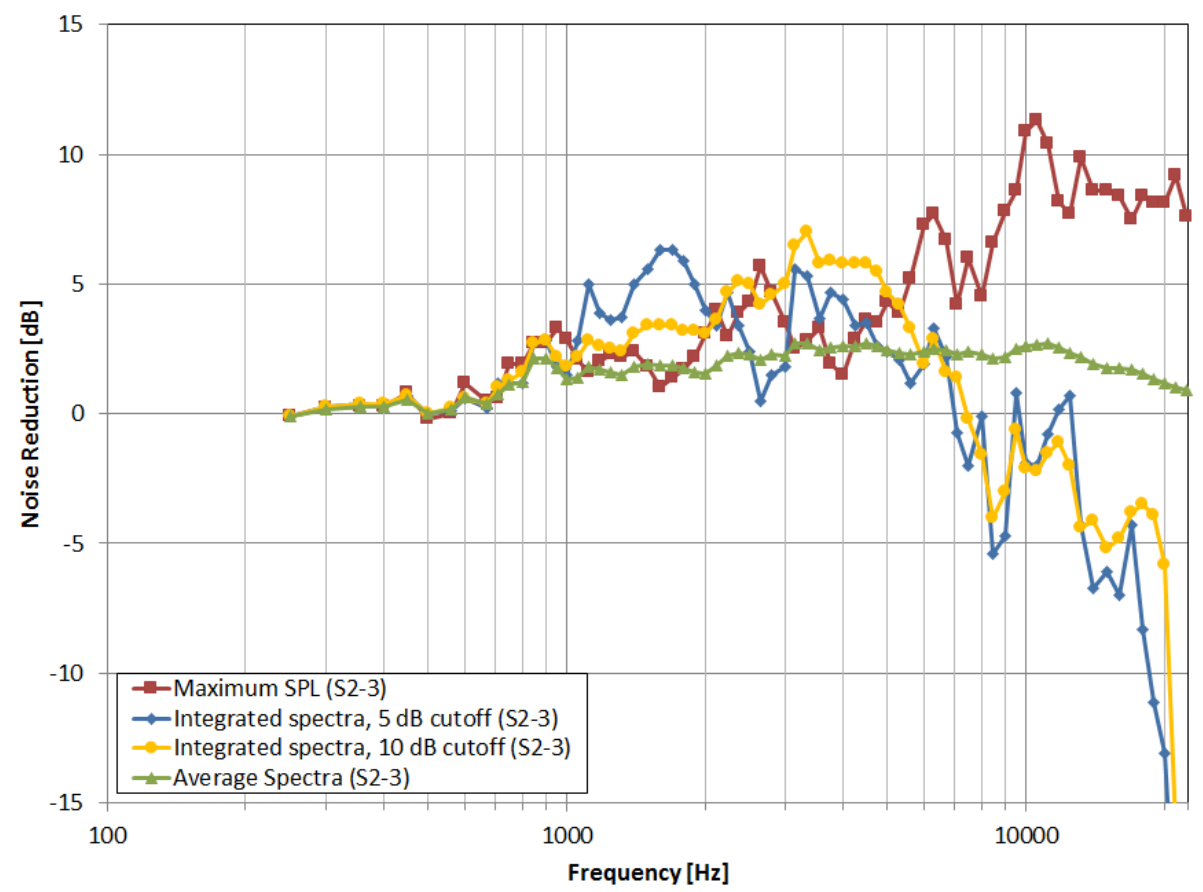

Figure 24. Flyover noise reduction from average spectra, integrated spectra, and maximum SPL in the maps for toboggan configuration at $13^{\circ}$ truck angle. Results in $1 / 12^{\text {th }}$ octave bands from sub-array S2-3. 

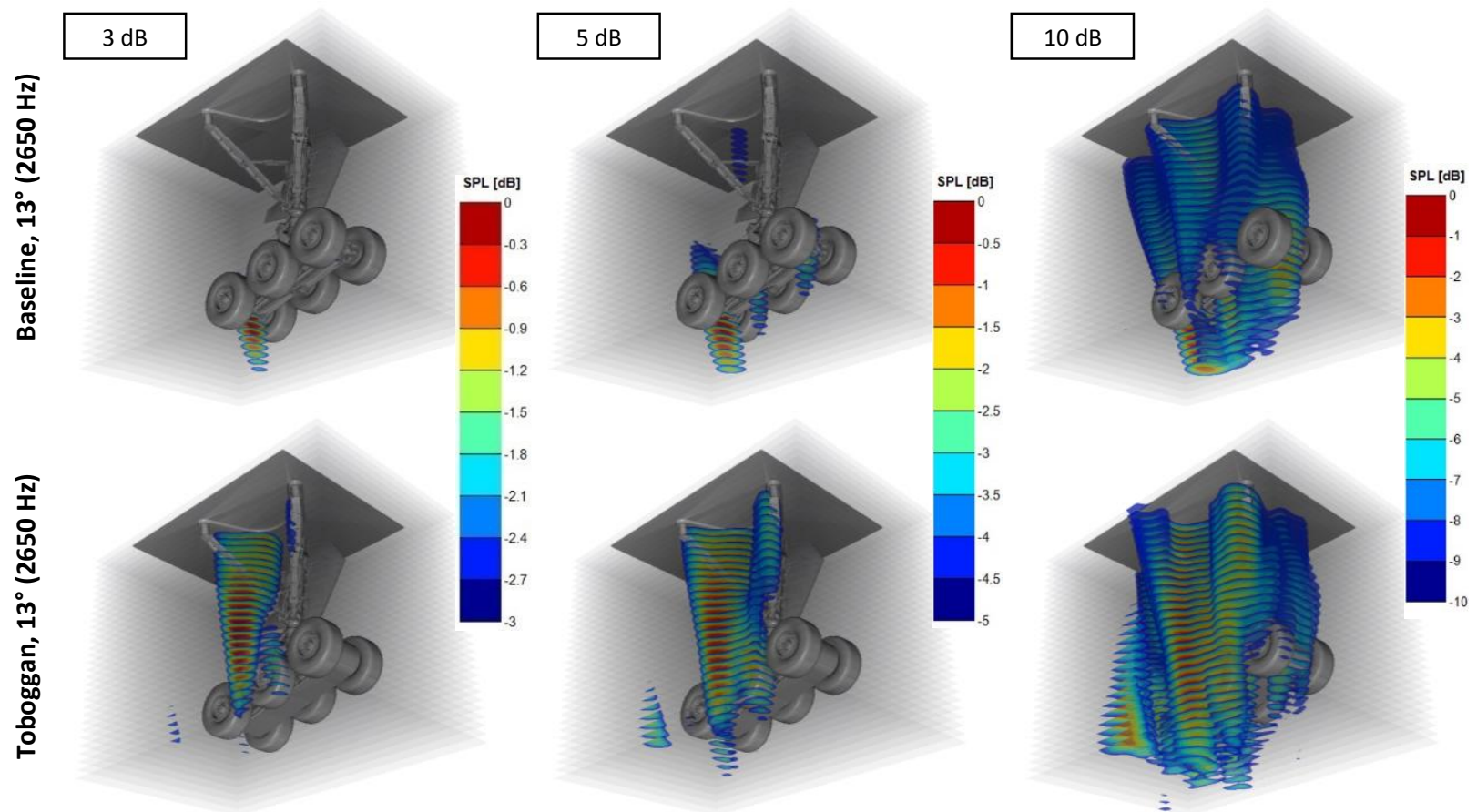

Figure 25. "3D" acoustic maps $\left(1 / 12^{\text {th }}\right.$ octave bands, $\left.2650 \mathrm{~Hz}\right)$ for $\mathrm{S} 2-3$ showing levels being integrated when a cutoff of 3,5 , or $10 \mathrm{~dB}$ is used. Contour levels in each figure set to the maximum in the grid. Toboggan peak level is $5 \mathrm{~dB}$ below the peak value in the baseline configuration.

Results for the toboggan at $0^{\circ}$ truck angle are shown in Figure 26. Unlike the $13^{\circ}$ cases, the toboggan clearly increases the noise (between 3 and $5 \mathrm{~dB}$ ) below $900 \mathrm{~Hz}$. A moderate noise increase (less than $1 \mathrm{~dB}$ ) is also observed between 1,000 and 1,400 Hz. Above these frequencies and below $16,000 \mathrm{~Hz}$, noise reductions of at least $\sim 1.5 \mathrm{~dB}$ are achieved based on the average spectra. The reduction obtained from consideration of the maximum SPL and visual inspection of the maps suggest that the $1.5 \mathrm{~dB}$ level mentioned above could be very conservative.

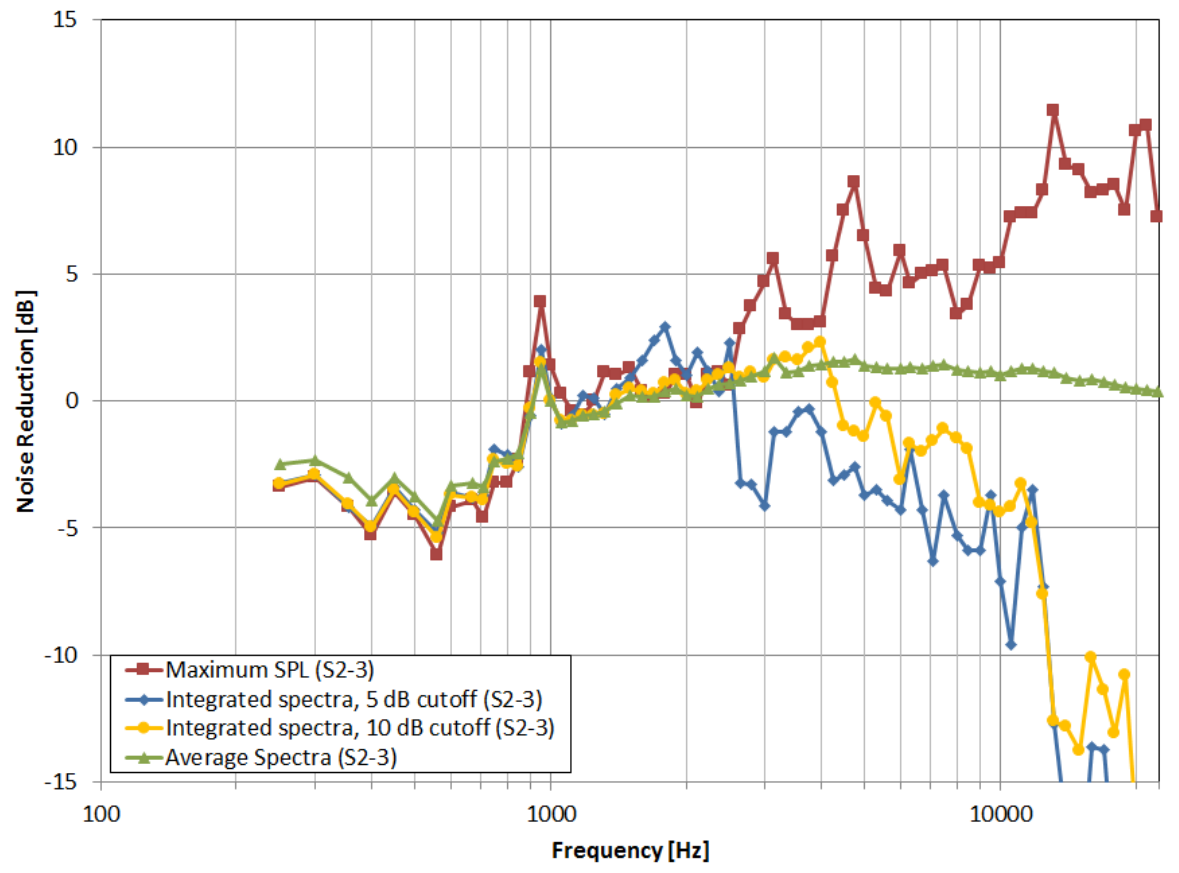

Figure 26. Flyover noise reduction from average spectra, integrated spectra, and maximum SPL in the maps for toboggan configuration at $0^{\circ}$ truck angle. Results in $1 / 12^{\text {th }}$ octave bands from sub-array S2-3. 
Figure 27 shows the corresponding results for the sideline measurements with a truck angle of $13^{\circ}$. Unlike the flyover measurements, where shielding effects might have played a significant role in the number of sources that are clearly visible, the sideline data show clear sources for most frequencies (for both baseline and toboggan configurations) and modest noise reduction levels. Therefore, the integrated values alone should provide adequate quantification. The results in Figure 27 suggest that a noise increase of up to $\sim 2 \mathrm{~dB}$ exists for frequencies below $700 \mathrm{~Hz}$. Noise reductions of about 1 to $3 \mathrm{~dB}$ are observed above this frequency. Notice that for some frequencies the noise reduction based on the maximum SPL is lower than that observed for the integrated and/or the average spectra. This suggests that the maximum level in a map has increased or that the number of sources or their relative levels have changed.

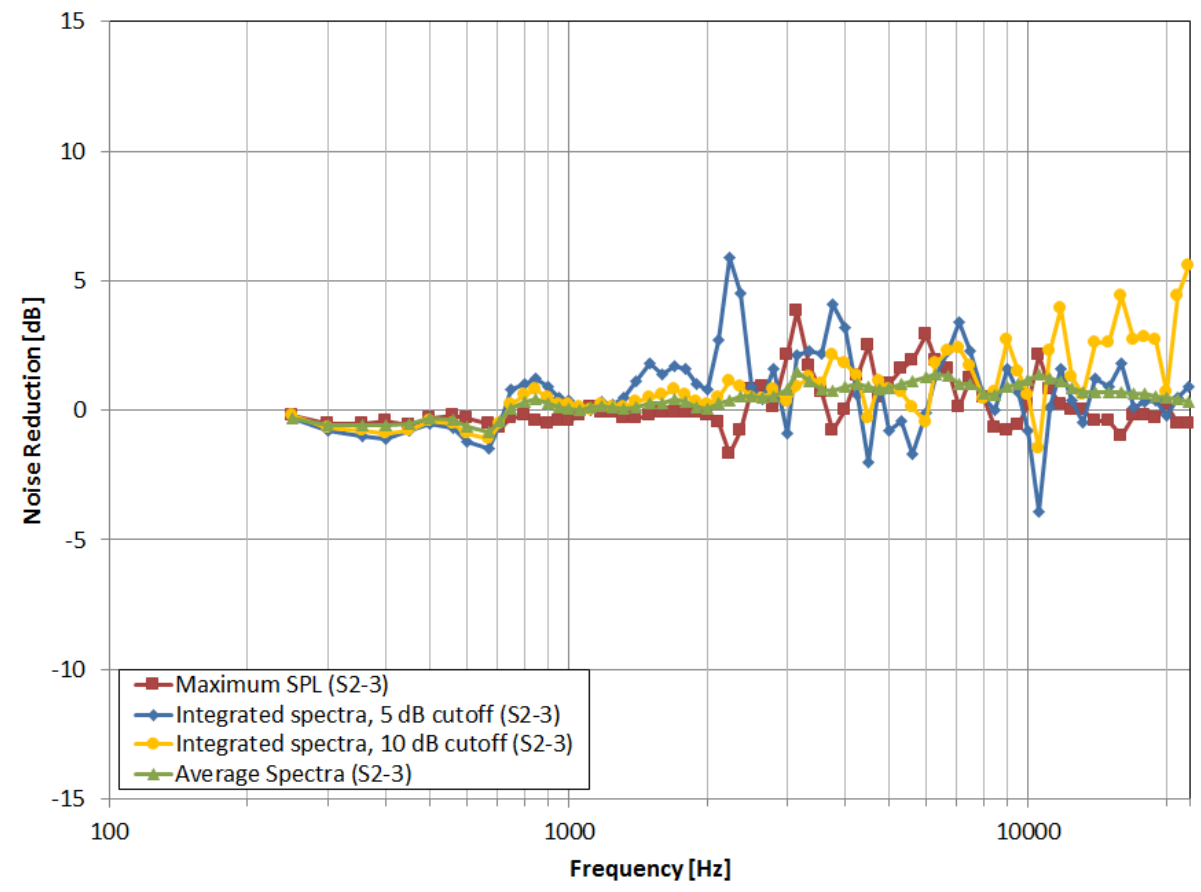

Figure 27. Sideline noise reduction from average spectra, integrated spectra, and maximum SPL in the maps for toboggan configuration at $13^{\circ}$ truck angle. Results in $1 / 12^{\text {th }}$ octave bands from sub-array S2-3.

Sample beamforming maps illustrating this behavior are presented in Figure 28 for a frequency of $2,240 \mathrm{~Hz}$. Although both maps use the same contour levels, note that the peak level for the toboggan configuration is $1.7 \mathrm{~dB}$ higher than the peak value for the baseline configuration. Similar results were observed for the $0^{\circ}$ cases shown in Figure 29. As seen in the figure, for these cases the toboggan increased the noise at most frequencies below $\sim 17,000 \mathrm{~Hz}$. Noise increments of up to $4 \mathrm{~dB}$ are observed at low frequencies.

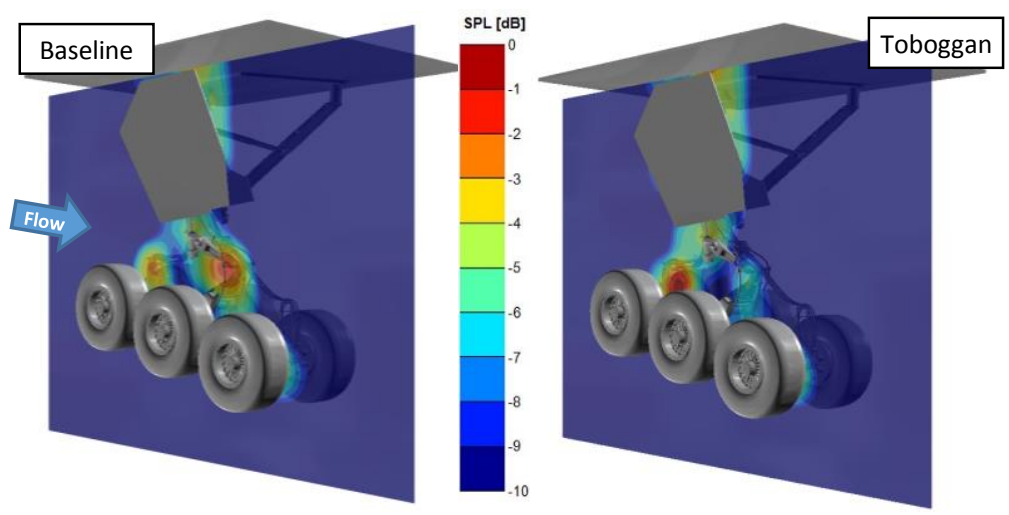

Figure 28. Comparison of sideline acoustic maps $(\mathbf{S 2 - 3 ,} 2240 \mathrm{~Hz})$ for baseline and toboggan configurations at $13^{\circ}$ truck angle. 


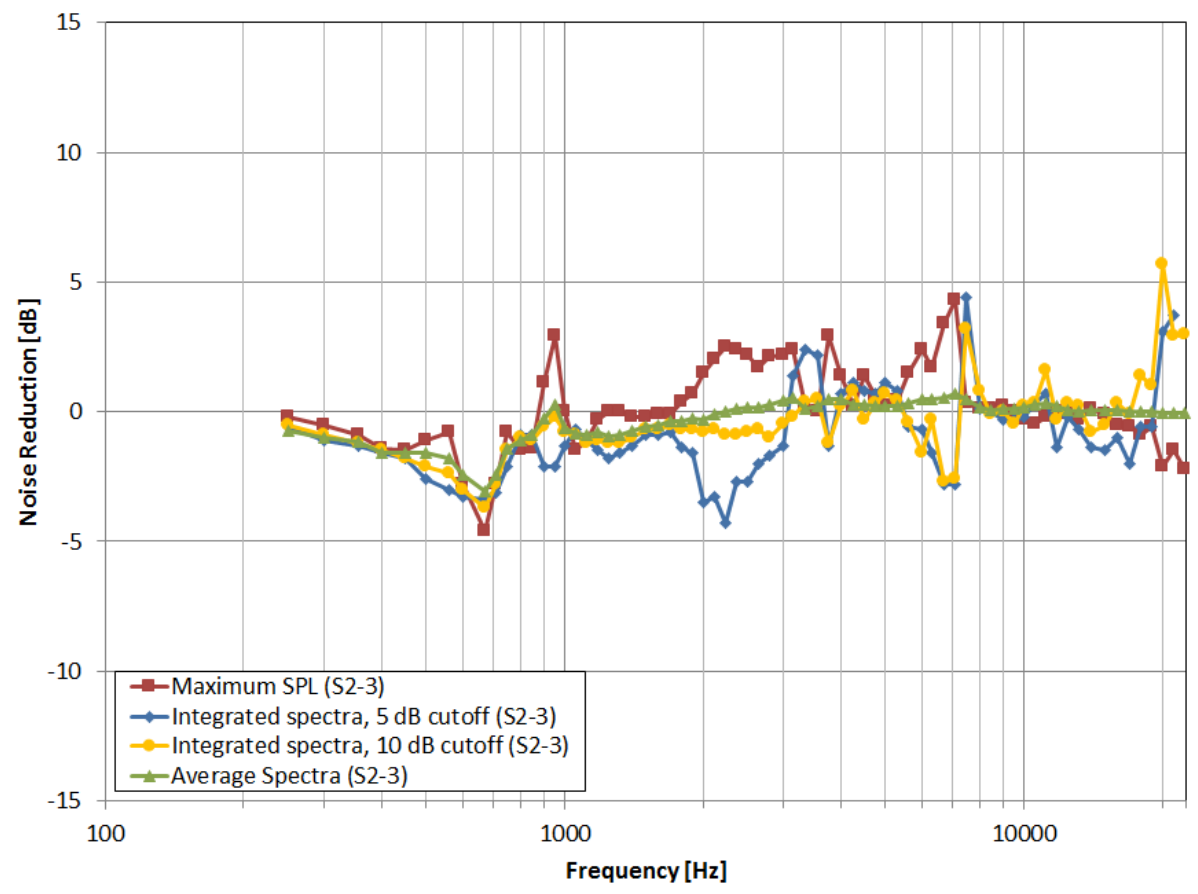

Figure 29. Sideline noise reduction from average spectra, integrated spectra, and maximum SPL in the maps for toboggan configuration at $0^{\circ}$ truck angle. Results in $1 / 12^{\text {th }}$ octave bands from sub-array $S 2-3$.

Since multiple sub-arrays were defined and used for beamforming purposes, the resulting maps can serve to identify differences in the sources visible with various array locations. This approach was of particular value for the flyover measurements where shielding effects from the installed toboggan were expected and previously deemed as the main reason for the differences in noise reduction observed between wind tunnel and flight tests. Integrating the maps from each sub-array also allows a rough quantification of directivity effects. Since identifying noise reduction is the main goal, the spectral levels presented below were not corrected for distance to the center of each sub-array.

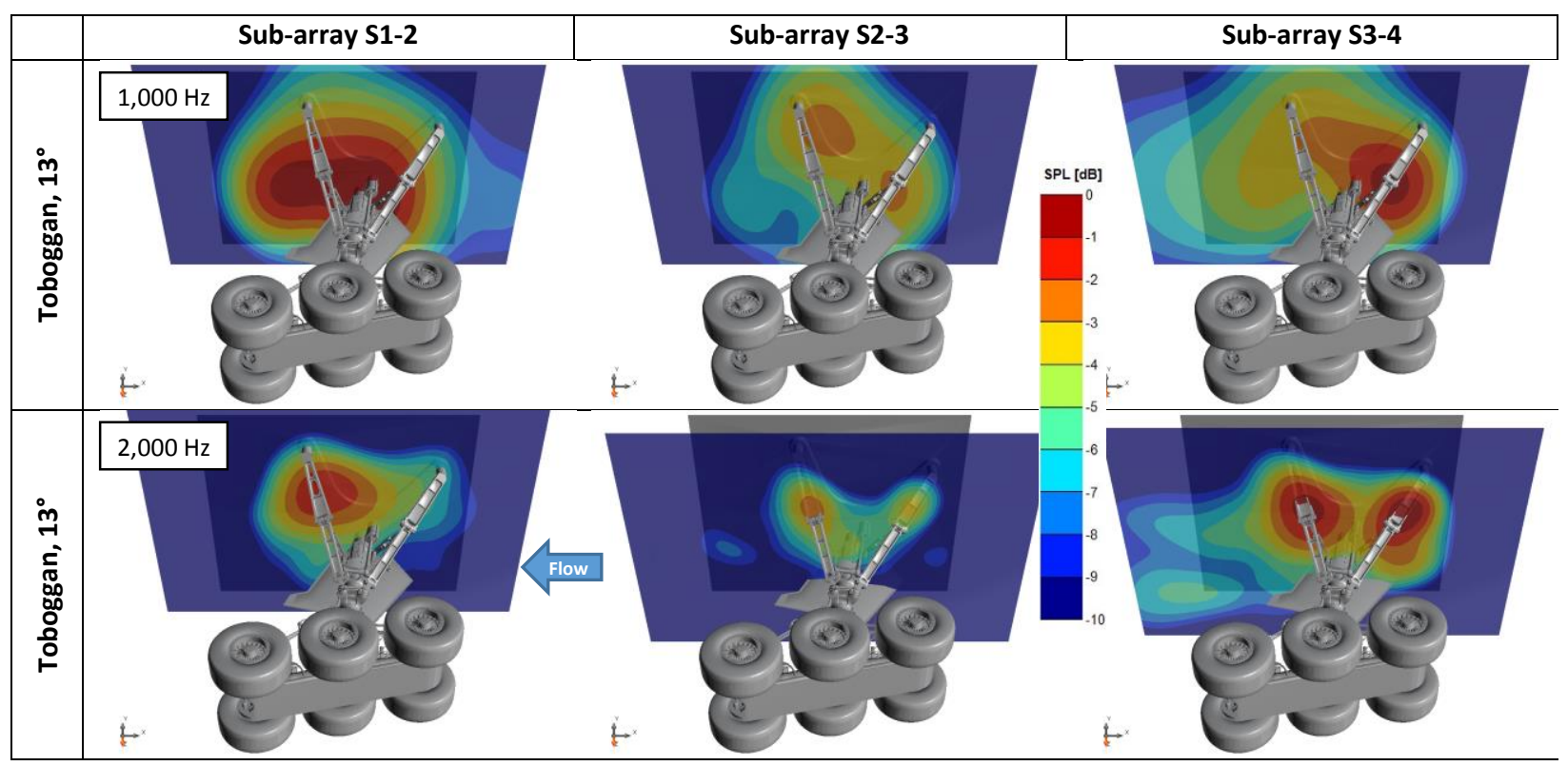

Figure 30. Sample 1/12 ${ }^{\text {th }}$ octave bands acoustic maps for sub-arrays S1-2, S2-3, and S3-4 for different landing gear configurations $(\mathrm{M}=\mathbf{0 . 1 7})$. 


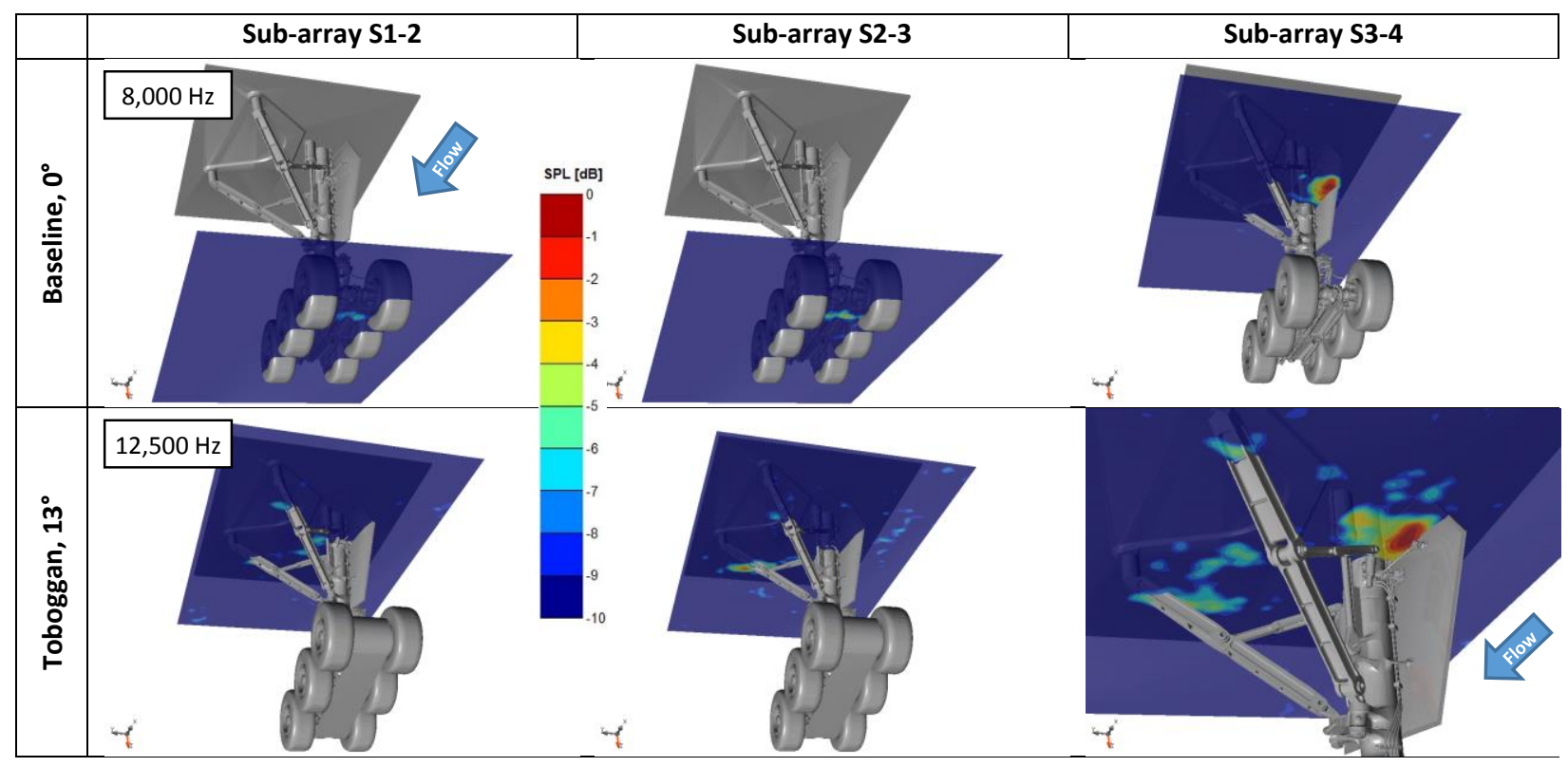

Figure 31. Sample 1/12 ${ }^{\text {th }}$ octave bands acoustic maps for sub-arrays S1-2, S2-3, and S3-4 for different landing gear configurations $(\mathrm{M}=\mathbf{0 . 1 7})$.

Figures 30 and 31 show a comparison of flyover acoustic maps for the three main sub-arrays analyzed (S1-2, S23 and S3-4), organized by column. The configuration and the $1 / 12^{\text {th }}$ octave band center frequency are also indicated for each row. The maximum level for maps in a row was set to the same peak value to facilitate the comparison. As can be seen, the characteristics of the acoustic maps (number of sources, their shape, relative levels, and location) can change significantly based on the directivity angle. The results show that S3-4 (located upstream of the model) can clearly identify sources in the main strut that could not be observed with the other arrays due to shielding or source directivity. For many frequencies, the peak levels obtained from the S3-4 sub-array are the loudest of all, suggesting the dominance of noise radiation in the forward direction.

Figure 32 shows the acoustic maps for the baseline and toboggan configurations using the sub-array S3-4. A comparison of these results to those presented in Figure 14 (obtained for S1-2 at the same frequency) indicates that different noise reduction levels would be obtained for each array location. A similar comparison for the sideline measurements is shown in Figure 33. In these maps, the peak levels from each sub-array do not vary as significantly as those for the flyover measurements. However, the relative levels between the sources at each frequency clearly change depending on the sub-array location. In this case, the gear door is shielding some of the sources on the main strut.
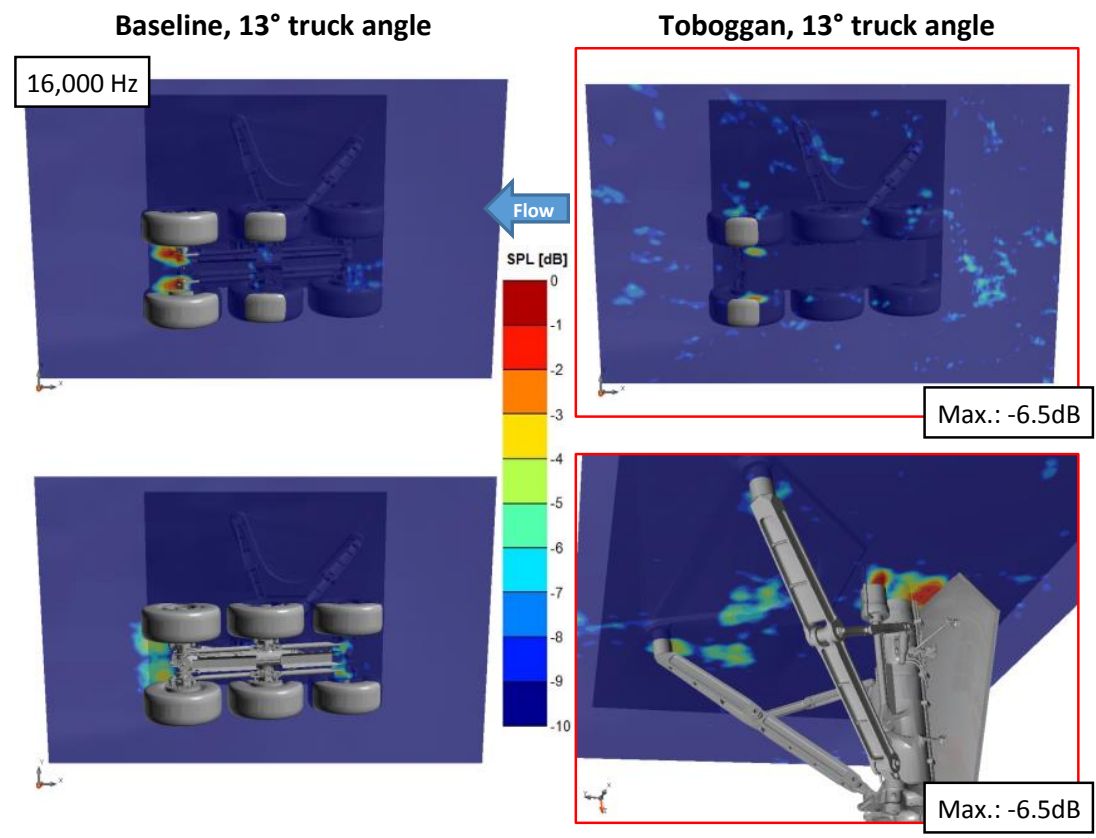

Figure 32. Comparison of $1 / 12^{\text {th }}$ octave bands acoustic maps (S3-4) for baseline and toboggan configurations. $M=0.17,1^{\circ}$ truck angle. 


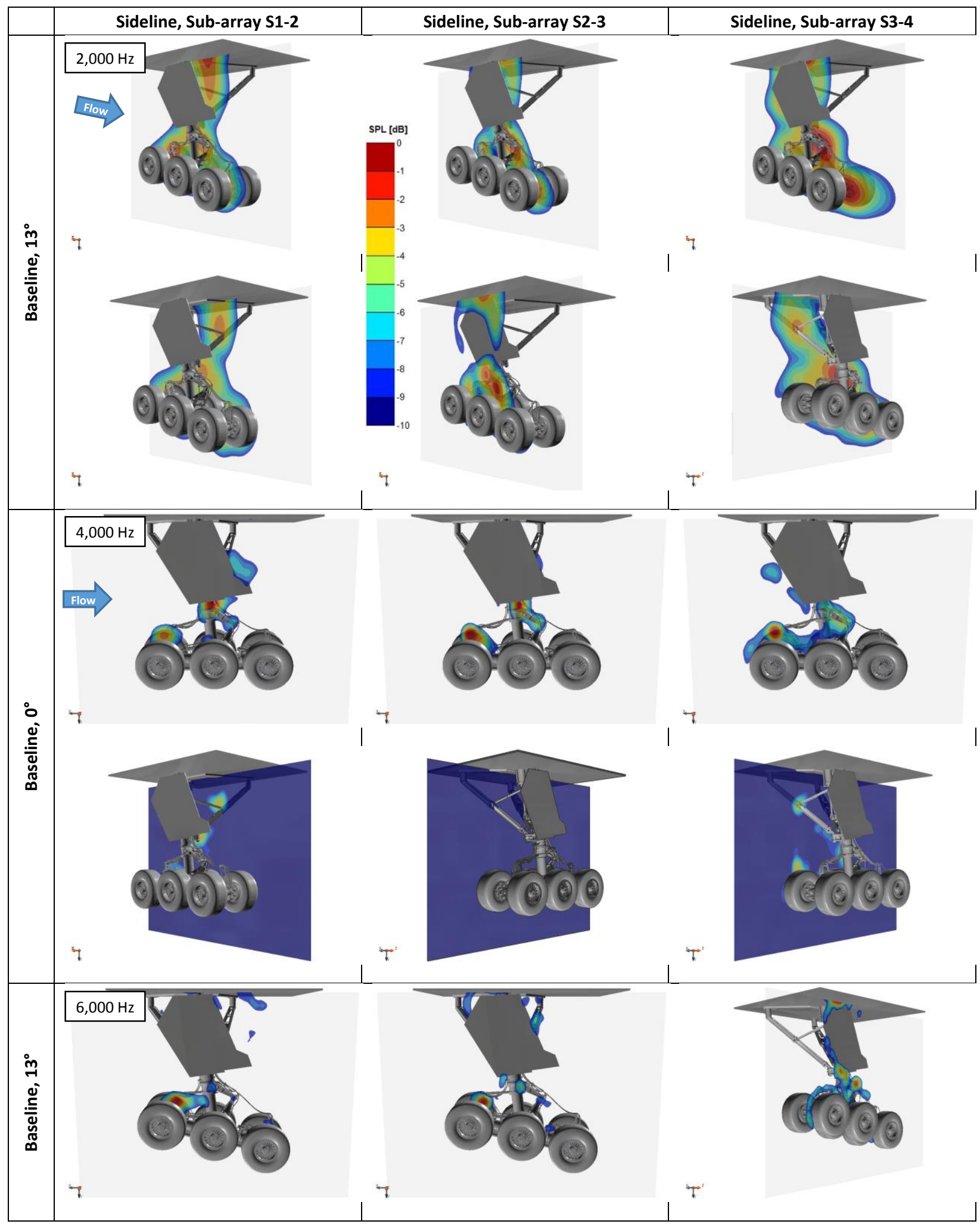

Figure 33. Sideline $1 / 12^{\text {th }}$ octave bands acoustic maps for sub-arrays S1-2, S2-3, and S3-4 for baseline landing gear at $0^{\circ}$ and $13^{\circ}$ truck angle $(\mathrm{M}=0.17)$. 
The integrated levels obtained with each sub-array for the baseline configuration are presented in Figure 34 for flyover and in Figure 35 for sideline measurements. Large level and shape variations are observed between the results of each sub-array. Thus, using results from a single array location only (like in previous tests) would ensure a mismatch between the noise reduction observed in a wind tunnel test and that observed on a flight test, where results are typically averaged over a range of directivity angles (along the flight path and normal to it) as the aircraft flies by the phased array.

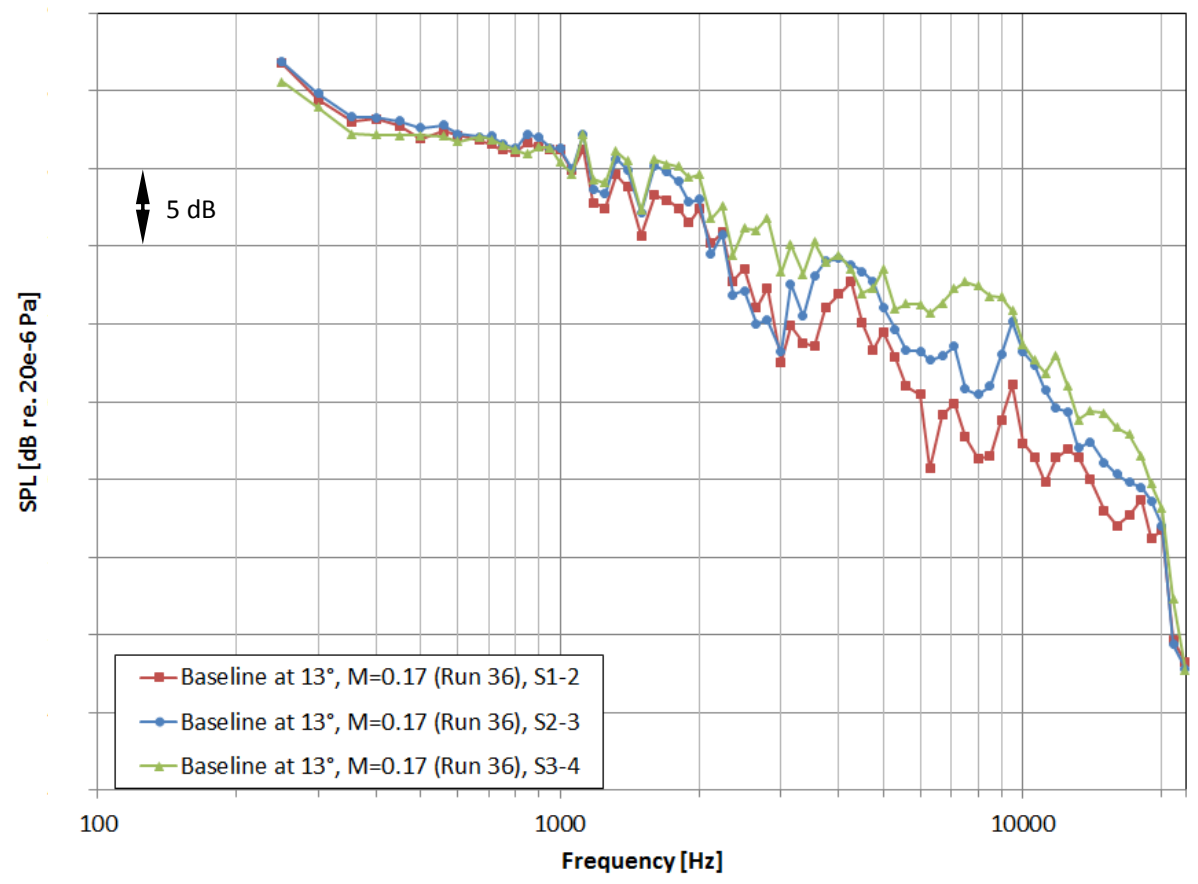

Figure 34. Flyover integrated spectra ( $5 \mathrm{~dB}$ cutoff) for baseline configuration at $13^{\circ}$ truck angle. Results in $1 / 12^{\text {th }}$ octave bands for three sub-arrays.

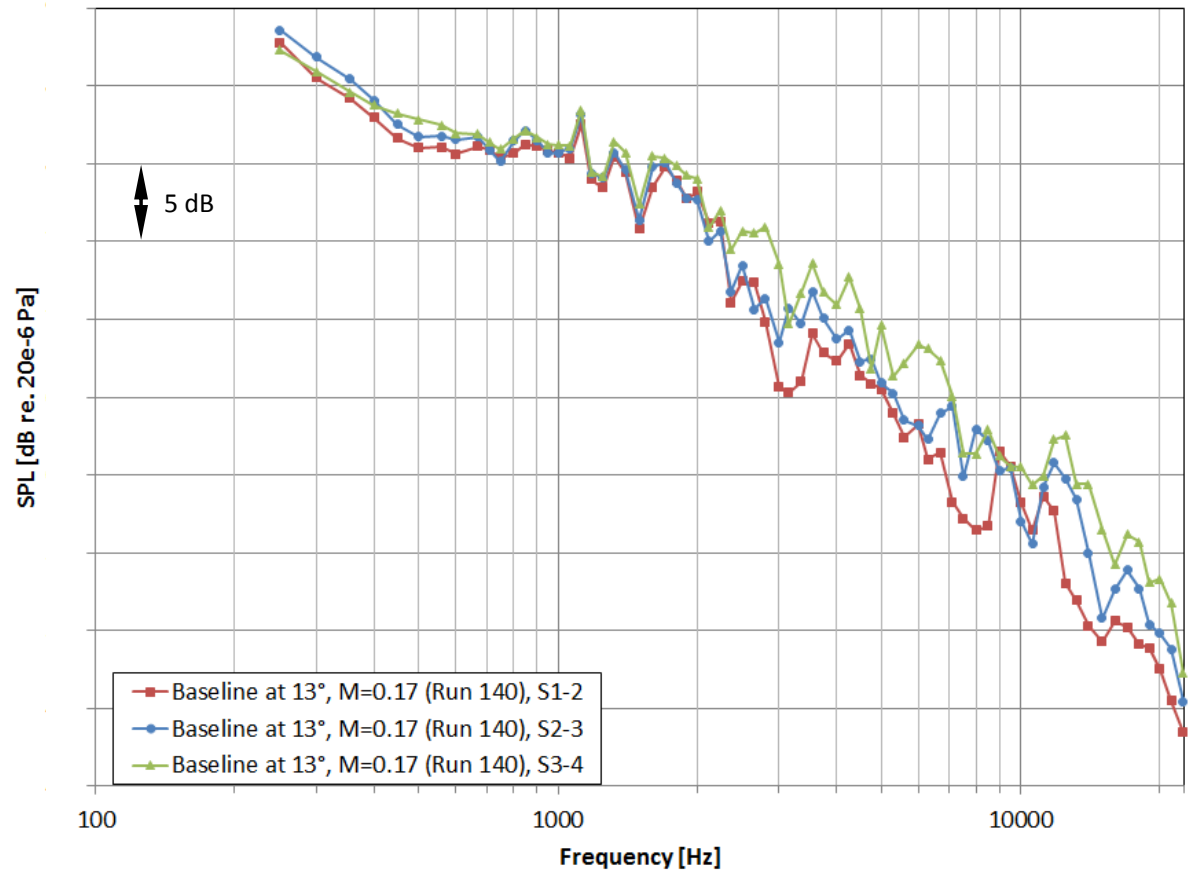

Figure 35. Sideline integrated spectra ( $5 \mathrm{~dB}$ cutoff) for baseline configuration at $13^{\circ}$ truck angle. Results in $1 / 12^{\text {th }}$ octave bands for three sub-arrays.

American Institute of Aeronautics and Astronautics 


\section{Concluding Remarks}

Aeroacoustic measurements of a 26\%-scale, Boeing 777-200 main landing gear model were performed in the Virginia Tech Stability Tunnel in its anechoic configuration. The phased array measurements were carried out using a newly available, large aperture, 251-element phased array that covers a wide directivity angle. Sub-arrays comprised of subsets of microphones were used to quantify the noise reduction potential of a toboggan fairing previously used in wind tunnel and flight tests. The use of a large array with multiple sub-arrays eliminated the need to repeat measurements with the array at different locations. This significantly reduced the test time (and cost) while also eliminating the potential of repeatability issues due to atmospheric conditions or setup changes (e.g., cabling locations, truck angle, etc.).

Noise source identification benefited from the improved array resolution that resulted from the large sub-array aperture. However, the large aperture also increased the number of sidelobes that combined with spurious sources within the beamformed grid, eventually hindering the accurate quantification of the integrated levels. Given the issues with the relatively low noise levels for the toboggan configuration and the spurious sources, a better approach for integrating this type of phased array data to obtain more accurate noise reduction levels would be desired. Average spectra and maximum levels in the maps were leveraged in an effort to accomplish this. The measurements indicated that, in some cases, noise reduction from the average spectra can aid in the task of determining the frequency range for which the integrated spectra yields accurate levels. This could reduce the need for visual inspection of each acoustic map, which was performed for all cases presented in this paper.

For the flyover measurements, sources that in previous tests were completely shielded by the truck were now clearly visible. With the toboggan fairing installed, the relatively low noise levels and the presence of contaminating sidelobes hindered the goal of better quantifying the noise reduction based on the integrated spectra levels, in particular at high frequencies. To overcome this deficiency, the average spectra and the maximum levels in the maps were used in an effort to improve estimation of the noise reduction levels that were achieved. In general, the estimated noise reduction from this test is slightly lower than that obtained from previous tests in the same tunnel. This lower estimate is mostly related to the larger aperture array that provides better insight into sources that were previously shielded by the truck.

The results presented here also suggest that a proper approach for comparing the noise reduction from wind tunnel tests to flight test results should involve modeling a "3D" directivity (possibly from the flyover and sideline data generated in this test) and simulating the flight path to obtain the expected levels at a microphone (or an array). Therefore, the resulting noise reduction would be a "weighted average" of the values obtained with the sideline and flyover sub-arrays.

The large body of data collected during this test campaign, as well as the flexibility of processing the array data using different sub-arrays (or implementing shading algorithms within them), provide a unique database for conducting comparative analyses with simulation results. In fact, preliminary comparisons of the acoustic maps to ongoing computations (to be published at a future date) are very encouraging.

\section{Acknowledgments}

This work was supported by the Environmentally Responsible Aviation (ERA) project under the Integrated Aviation Systems Program (IASP) of NASA. The authors would like to thank the Virginia Tech wind tunnel directors and its personnel.

\section{References}

${ }^{1}$ Dobrzynski, W., “Almost 40 Years of Airframe Noise Research: What Did We Achieve, ” J. Aircraft, Vol. 47, No.2, MarchApril 2010.

${ }^{2}$ Michel, U., Barsikow, B., Helbig, J., Hellmig, M., and Schüttpelz, M., "Flyover Noise Measurements on Landing Aircraft with a Microphone Array," AIAA Paper 98-2336, May 1998.

${ }^{3}$ Piet, J.-F., Elias, G., and Lebigot, P., "Localization of Acoustic Sources from a Landing Aircraft with a Microphone Array," AIAA Paper 99-1811, May 1999.

${ }^{4}$ Stoker, R., Guo, Y., Streett, C., and Burnside, N., "Airframe Noise Source Locations of a 777 Aircraft in Flight and Comparisons with Past Model Tests,” AIAA Paper 2003-3232, May 2003.

${ }^{5}$ Brusniak, L., Underbrink, J. R., and Stoker, R. W., “Acoustic Imaging of Aircraft Noise Sources Using Large Aperture Phased Arrays," AIAA Paper 2006-2715, May 2006.

${ }^{6}$ Elkoby, R., Brusniak, L., Stoker, R., Khorrami, M. R., Abeysinghe, A., and Moe, J. W., "Airframe Noise Results from the QTD II Flight Test Program,” AIAA Paper 2007-3457, May 2007.

${ }^{7}$ Khorrami, M. R. and Mineck, R. E., "Towards Full Aircraft Airframe Noise Prediction: Detached Eddy Simulations,” AIAA Paper 2014-2480, June 2014. 
${ }^{8}$ Khorrami, M. R., Fares, E., and Casalino, D., "Towards Full-Aircraft Airframe Noise Prediction: Lattice-Boltzmann Simulations," AIAA Paper 2014-2481, June 2014.

${ }^{9}$ Fares, E., Casalino, D., and Khorrami, M. R., "Evaluation of Airframe Noise Reduction Concepts via Simulations using a Lattice-Boltzmann Approach,” AIAA Paper 2015-2988, June 2015.

${ }^{10}$ Khorrami, M. R., and Fares, E., "Simulation-Based Airframe Noise Prediction of a Full-Scale Full Aircraft," Paper to be presented at the 22nd AIAA/CEAS Aeroacoustics Conference in Lyon, France, May-June 2016.

${ }^{11}$ Khorrami, M. R., Fares, E., Duda, B., and Hazir, A., "Full Scale Evaluation of Airframe Noise Reduction Concepts via Computational Simulations," Paper to be presented at the 22nd AIAA/CEAS Aeroacoustics Conference in Lyon, France, MayJune 2016.

${ }^{12}$ Fares, E., Duda, B., and Khorrami, M. R., "Airframe Noise Prediction of a Detailed Full Aircraft in Model and Full Scale Using a Lattice Boltzmann Approach," Paper to be presented at the 22nd AIAA/CEAS Aeroacoustics Conference in Lyon, France, May-June 2016.

${ }^{13}$ Jaeger, S. M., Burnside, N. J., Soderman, P. T., Horne,W. C., and James, K. D., "Microphone Array Assessment of an Isolated, 26\%-Scale, High Fidelity Landing Gear,” AIAA Paper 2002-2410, June 2002.

${ }^{14}$ Ravetta, P. A., Burdisso, R. A., and Ng, W. F., "Wind Tunnel Aeroacoustic Measurements of a 26\%-scale 777 Main Landing Gear Model," AIAA Paper 2004-2885, May 2004.

${ }^{15}$ Ravetta, P. A., Burdisso, R. A., Ng, W. F., Khorrami, M. R., and Stoker, R. W., "Screening of Potential Noise Control Devices at Virginia Tech for QTD II Flight Test," AIAA Paper 2007-3455, May 2007.

${ }^{16}$ Horne, W. C., James, K. D., Arledge, T. K., Soderman, P. T., Burnside, N., and Jaeger, S. M., "Measurements of 26\%-scale 777 Airframe Noise in the NASA Ames 40- by 80 Foot Wind Tunnel," AIAA Paper 2005-2810, May 2005.

${ }^{17}$ Remillieux, M. C., Camargo, H. E., Ravetta, P. A., Burdisso, R. A., and Ng, W. F., "Novel Kevlar-Walled Wind Tunnel for Aeroacoustic Testing of a Landing Gear," AIAA J., Vol. 46, No. 7, pp. 1631-1639, July 2008.

${ }^{18}$ Underbrink, J. R, "Pletharrays for Aeroacoustic Phased Array Applications," AIAA Paper 2015-2978, June 2015.

${ }^{19}$ Mueller T. (ed.), Aeroacoustic Measurements, Springer, 2002. ISBN 3-540-41757-5.

${ }^{20}$ Devenport W.J., Burdisso R.A., Borgoltz A., Ravetta P.A., Barone M.F., Brown K.A., and Morton M.A., "The Kevlar-walled anechoic wind tunnel, " Journal of Sound and Vibration, Vol. 332, No. 17, August 2013, pp. 3971-3991 